\title{
ON THE CONTROLLABILITY OF THE 2-D \\ INCOMPRESSIBLE NAVIER-STOKES EQUATIONS WITH THE NAVIER SLIP BOUNDARY CONDITIONS
}

\author{
JEAN-MICHEL CORON
}

\begin{abstract}
For boundary or distributed controls, we get an approximate controllability result for the Navier-Stokes equations in dimension 2 in the case where the fluid is incompressible and slips on the boundary in agreement with the Navier slip boundary conditions.
\end{abstract}

Keywords: Controllability, Navier-Stokes equations, Navier slip boundary conditions.

\section{INTRODUCTION}

Let $\Omega$ be a bounded nonempty connected open subset of $\mathbb{R}^{2}$ of class $C^{\infty}$. Let $\Gamma^{\#}$ be an open subset of $\Gamma:=\partial \Omega$ and let $\Omega^{\#}$ be an open subset of $\Omega$. We assume that

$$
\Gamma^{\#} \cup \Omega^{\#} \neq \emptyset .
$$

We denote by $n$ the outward unit normal vector field on $\Gamma$ and by $\tau$ the unit tangent vector field on $\Gamma$ such that $(\tau, n)$ is a direct basis of $\mathbb{R}^{2}$. The set $\Gamma^{\#}$ is the part of the boundary and $\Omega^{\#}$ is the part of the domain $\Omega$ on which the controls acts. The fluid that we consider is incompressible so that the velocity field $y$ satisfies

$$
\operatorname{div} y=0 \text {. }
$$

On the part of the boundary $\Gamma \backslash \Gamma^{\#}$ where there is no control the fluid slips; it satisfies

$$
y \cdot n=0 \text { on } \Gamma \backslash \Gamma^{\#}
$$

and the Navier slip boundary condition [24]

$$
\bar{\sigma} y \cdot \tau+(1-\bar{\sigma}) n^{i}\left(\frac{\partial y^{i}}{\partial x^{j}}+\frac{\partial y^{j}}{\partial x^{i}}\right) \tau^{j}=0 \text { on } \Gamma \backslash \Gamma^{\#}
$$

where $\bar{\sigma}$ is a constant in $[0,1), n=\left(n^{1}, n^{2}\right), \tau=\left(\tau^{1}, \tau^{2}\right)$, and where we have used the usual summation convention. Note that the classical no-slip condition, due to Stokes,

$$
y=0
$$

corresponds to the case $\bar{\sigma}=1$, which is not considered here. The slip boundary condition (1.3) with $\bar{\sigma}=0$ corresponds to the case where there

Centre de Mathématiques et Leurs Applications, École Normale Supérieure de Cachan et CNRS URA 1611, 61 Av. du Pdt Wilson, 94235 Cachan Cedex, France. E-mail address: Jean-Michel .Coronecmla.ens-cachan.fr.

Received by the journal January 5, 1996. Accepted for publication April 15, 1996.

This work was partially supported by the GDR "Automatique" of the CNRS. 
the fluid slips on the wall without friction. It is the appropriate physical model for some flow problems; see [16] for example. The case $\bar{\sigma} \in(0,1)$ corresponds to a case where there the fluid slips on the wall with friction; it is also used in models of turbulence with rough walls; see, e.g., [17]. Note that in [3] F. Coron has derived rigorously the slip boundary condition (1.3) from the boundary condition at the kinetic level (Boltzmann equation) for compressible fluids. Let us also recall that C. Bardos, F. Golse, and D. Levermore have derived in [2] the incompressible Navier-Stokes equations from a Boltzmann equation.

Let us point out that, using (1.2), one sees that (1.3) is equivalent to

$$
\sigma y \cdot \tau+\operatorname{curl} y=0 \text { on } \Gamma \backslash \Gamma^{\#}
$$

with $\sigma \in C^{\infty}(\Gamma ; \mathbb{R})$ defined by

$$
\sigma(x)=\frac{2(1-\bar{\sigma}) \kappa(x)-\bar{\sigma}}{1-\bar{\sigma}}, \forall x \in \Gamma,
$$

where $\kappa$ is the curvature of $\Gamma$ defined through the relation $\frac{\partial n}{\partial \tau}=\kappa \tau$. In fact we will not use this particular character of (1.5) in our considerations; Theorem 1.1 below holds for any $\sigma \in C^{\infty}(\Gamma ; \mathbb{R})$.

The problem of approximate controllability we consider is the following one: let $T>0$, let $y_{0}$ and $y_{1}$ in $C^{\infty}\left(\bar{\Omega} ; \mathbb{R}^{2}\right)$ be such that

$$
\begin{gathered}
\operatorname{div} y_{0}=0 \text { in } \bar{\Omega}, \\
\operatorname{div} y_{1}=0 \text { in } \bar{\Omega}, \\
y_{0} \cdot n=0 \text { on } \Gamma \backslash \Gamma^{\#}, \\
y_{1} \cdot n=0 \text { on } \Gamma \backslash \Gamma^{\#}, \\
\sigma y_{0} \cdot \tau+\operatorname{curl} y_{0}=0 \text { on } \Gamma \backslash \Gamma^{\#}, \\
\sigma y_{1} \cdot \tau+\operatorname{curl} y_{1}=0 \text { on } \Gamma \backslash \Gamma^{\#} .
\end{gathered}
$$

We ask whether there exist $y \in C^{\infty}\left(\bar{\Omega} \times[0, T] ; \mathbb{R}^{2}\right)$ and $p \in C^{\infty}(\bar{\Omega} \times[0, T] ; \mathbb{R})$ such that

$$
\begin{gathered}
\frac{\partial y}{\partial t}-\Delta y+(y \cdot \nabla) y+\nabla p=0 \text { in }\left(\bar{\Omega} \backslash \Omega^{\#}\right) \times[0, T], \\
\operatorname{div} y=0 \text { in } \bar{\Omega} \times[0, T], \\
y \cdot n=0 \text { on }\left(\Gamma \backslash \Gamma^{\#}\right) \times[0, T], \\
\sigma y \cdot \tau+\operatorname{curl} y=0 \text { on }\left(\Gamma \backslash \Gamma^{\#}\right) \times[0, T], \\
y(\cdot, 0)=y_{0} \text { in } \bar{\Omega}
\end{gathered}
$$

and, in an appropriate topology, we have

$$
y(\cdot, T) \text { is "close" to } y_{1} \text {. }
$$

That is to say, starting with the initial data $y_{0}$ for the Navier-Stokes equations, we ask whether there are solutions which, at a fixed time $T$, approach arbitrarily closely to the given velocity field $y_{1}$. 
Note that (1.12) to (1.16) have many solutions. In order to have uniqueness one needs to add extra conditions. These extra conditions are the controls. Various possible controls can be considered. For example, a possible choice for the controls is

$$
\begin{gathered}
y \cdot n \text { on } \Gamma^{\#} \times[0, T], \\
\sigma y \cdot \tau+\operatorname{curl} y \text { on } \Gamma^{\#} \times[0, T], \\
\frac{\partial y}{\partial t}-\Delta y+(y \cdot \nabla) y+\nabla p \text { in } \Omega^{\#} \times[0, T] .
\end{gathered}
$$

More precisely, let $y_{s} \in C^{\infty}\left(\bar{\Omega} \times[0, T] ; \mathbb{R}^{2}\right)$ and $p_{s} \in C^{\infty}(\bar{\Omega} \times[0, T] ; \mathbb{R})$ be such that (1.12) to (1.16) hold for $(y, p)=\left(y_{s}, p_{s}\right)$. Let us consider the following Cauchy problem: find $y \in C^{\infty}\left(\bar{\Omega} \times[0, T] ; \mathbb{R}^{2}\right)$ and $p \in C^{\infty}(\bar{\Omega} \times[0, T] ; \mathbb{R})$ such that (1.12) to (1.16) hold and

$$
\begin{aligned}
y \cdot n & =y_{s} \cdot n \text { on } \Gamma^{\#} \times[0, T] \\
\sigma y \cdot \tau+\operatorname{curl} y & =\sigma y_{s} \cdot \tau+\operatorname{curl} y_{s} \text { on } \Gamma^{\#} \times[0, T] \\
\frac{\partial y}{\partial t}-\Delta y+(y \cdot \nabla) y+\nabla p & =\frac{\partial y_{s}}{\partial t}-\Delta y_{s}+\left(y_{s} \cdot \nabla\right) y_{s}+\nabla p_{s} \text { in } \Omega^{\#} \times[0, T]
\end{aligned}
$$

has, up to an arbitrary function depending only on time added to $p$, one and only one solution which is $(y, p)=\left(y_{s}, p_{s}\right)$. One can also use for the control (1.18), (1.20), and curl $y$ on $\Gamma^{\#} \times[0, T]$. Another possibility for the control is $(1.20)$ and $y$ on $\Gamma^{\#} \times[0, T]$.

Let $d \in C^{0}(\bar{\Omega} ; \mathbb{R})$ be defined by

$$
d(x)=\operatorname{dist}(x, \Gamma)=\operatorname{Min}\left\{\left|x-x^{\prime}\right| ; x^{\prime} \in \Gamma\right\} .
$$

Our controllability result is

Theorem 1.1. Let $T>0$, let $y_{0}$ and $y_{1}$ in $C^{\infty}\left(\bar{\Omega}, \mathbb{R}^{2}\right)$ be such that (1.6), (1.7), (1.8), (1.9), (1.10), and (1.11) hold. Then, there exist a sequence $\left(y^{k} ; k \in \mathbb{N}\right)$ of maps in $C^{\infty}\left(\bar{\Omega} \times[0, T] ; \mathbb{R}^{2}\right)$ and a sequence $\left(p^{k} ; k \in \mathbb{N}\right)$ of functions in $C^{\infty}(\bar{\Omega} \times[0, T] ; \mathbb{R})$ such that, for all $k \in \mathbb{N}$, (1.12), (1.13), (1.14), (1.15), and (1.16) hold for $y=y^{k}$ and $p=p^{k}$ and such that, as $k \rightarrow+\infty$,

$$
\begin{gathered}
\int_{\Omega} d^{\mu}\left|y^{k}(\cdot, T)-y_{1}\right| \rightarrow 0, \forall \mu>0, \\
\left|y^{k}(\cdot, T)-y_{1}\right|_{W^{-1, \infty}(\Omega)} \rightarrow 0,
\end{gathered}
$$

and, for all compact $K$ included in $\Omega \cup \Gamma^{\#}$,

$$
\left|y^{k}(\cdot, T)-y_{1}\right|_{L^{\infty}(K)}+\left|\operatorname{curl} y^{k}(\cdot, T)-\operatorname{curl} y_{1}\right|_{L^{\infty}(K)} \rightarrow 0 .
$$

In this theorem, and throughout all this paper, $W^{-1, \infty}(\Omega)$ denotes the usual Sobolev space of first derivatives of functions in $L^{\infty}(\Omega)$ and ||$_{W^{-1, \infty}(\Omega)}$ one of it's usual norms, for example the norm given in [1, Section 3.10].

REMARK 1.2. a) The question of the approximate controllability of the

Navier-Stokes equations for incompressible fluids has been raised by J.-L Lions in [20] and [21] for the no-slip boundary condition $y=0$ on $\left(\Gamma \backslash \Gamma^{\#}\right) \times[0, T]$. 
b) Note that $(1.21),(1.22)$, and (1.23) are not strong enough to imply

$$
\left|y^{k}(\cdot, T)-y_{1}\right|_{L^{2}(\Omega)} \rightarrow 0
$$

But, in the special case where $\sigma=0$ in $\Gamma \backslash \Gamma^{\#}$, Theorem 1.1 still holds even if one requires also (1.24); see Remark 2.4 below.

c) Let us point out that, if $\Gamma^{\#}=\Gamma$, there is, of course, no difference between the slip case and the no-slip case. Theorem 1.1 is, up to our knowledge, new even if $\Gamma^{\#}=\Gamma$; in this special case the proof of Theorem 1.1 can be strongly simplified. If $\Gamma^{\#}=\Gamma$, we get from (1.23) that $y^{k}(\cdot, T) \rightarrow y$ in the Sobolev space $H^{1}(\Omega)$ as $k \rightarrow \infty$, i.e. we prove approximate controllability in $H^{1}(\Omega)$. So we give a positive answer to J.-L. Lions's conjecture [20]-[21] in dimension 2 when $\Gamma^{\#}=\Gamma$. Similarly, if, for some $\varepsilon_{0}>0,\left\{x \in \Omega ; d(x)<\varepsilon_{0}\right\} \subset \Omega^{\#}$, we get the same result, i.e., approximate controllability in $H^{1}(\Omega)$ for the Navier boundary condition and for the no-slip boundary condition. To see it, let, for $\varepsilon^{\prime} \in\left(0, \varepsilon_{0} / 2\right), \Omega^{\prime}=\left\{x \in \Omega ; d(x)>\varepsilon^{\prime}\right\}$, let $\Gamma^{\prime}=\partial \Omega^{\prime}$, let $\Gamma_{0}^{\prime}, \ldots, \Gamma_{i}^{\prime}, \ldots, \Gamma_{g}^{\prime}$ be the connected components of $\Gamma$, and let $\Gamma^{\# \prime}$ be the union of the $\Gamma_{i}^{\prime}$ such that Min $\left\{\operatorname{dist}\left(x, \Gamma^{\#}\right) ; x \in \Gamma_{i}^{\prime}\right\} \leqslant \varepsilon^{\prime}$. Apply Theorem 1.1 with $\Omega^{\prime}$ for $\Omega, \Omega^{\prime} \cap \Omega^{\#}$ for $\Omega^{\#}, \Gamma^{\# \prime}$ for $\Gamma^{\#}$, and $\varepsilon^{\prime}>0$ small enough. Then, for the $\left(y^{k}, p^{k}\right)$ given by Theorem 1.1, extend $p^{k}$ to all of $\bar{\Omega}$, modify in a suitable way $y^{k}$ in $\left\{x \in \overline{\Omega^{\prime}} ; d(x)<2 \varepsilon^{\prime}\right\}$ and finally extend in a suitable way the new $y^{k}$ to all of $\bar{\Omega}$.

d) Of course, by density, Theorem 1.1 still holds if $y_{1}$ is only of class $C^{1}$. Moreover, as it will follow from our proof, one can also assume less regularity on $y_{0}$ if one requires only that $\left(y^{k}, p^{k}\right)$ are of class $C^{\infty}$ on $\bar{\Omega} \times(0, T]$. See Remark 3.1 below for more details.

e) E. Fernández-Cara and J. Real in [11] and E. Fernández-Cara and M. González-Burgos in [10] have proved that, for 2-D and 3-D incompressible fluids, the linear space spanned by the $y(\cdot, T)$ such that, for some $p: \bar{\Omega} \times[0, T] \rightarrow \mathbb{R}$, one has (1.12), (1.13), (1.16), and the no-slip boundary condition, is dense, with respect to the $L^{2}$-norm in the set of $y_{1}: \bar{\Omega} \rightarrow \mathbb{R}^{2}$ satisfying (1.7) and $y_{1}=0$ on $\Gamma \backslash \Gamma^{\#}$.

f) A.V. Fursikov and O.Yu Imanuvilov have proved in [13] [14] that, if $\Gamma^{\#}=\Gamma$, then one has exact zero controllability in large time, i.e., for any $y_{0}$ satisfying (1.6), there exist $T>0, y$ and $p$ satisfying (1.12) to (1.16) and $y(\cdot, T)=0$. In [15], they have recently obtained the same result in the more general situation where $\sigma=0$ in $\Gamma \backslash \Gamma^{\#}$. Again if $\Gamma^{\#}=\Gamma, A . V$. Fursikov has proved in [12] the exact zero controllability in large time in dimension 3.

g) In [9] C. Fabre has obtained, in every dimension, an approximate controllability of two natural "cut off" Navier-Stokes equations (with the no-slip boundary condition).

As in our proof of the controllability of the 2-D Euler equations of incompressible perfect fluids $[7,8]$, the strategy of the proof of Theorem 1.1 relies on a method described in [5] and [6] under the name of "the return method". This was introduced in [4] for a stabilization problem. Roughly speaking it consists in looking for $(\bar{y}, \bar{p})$ such that $(1.12)$ to (1.15) hold with Esaim: CoCV, MaY 1996, VOL. 1, PP. 35-75. 
$y=\bar{y}, p=\bar{p}$,

$$
\bar{y}(\cdot, 0)=\bar{y}(\cdot, T)=0 \text { in } \bar{\Omega},
$$

and such that the linearized control system around $(\bar{y}, \bar{p})$ has a controllability in a "good" sense. With such a $(\bar{y}, \bar{p})$ one may hope that there exists $(y, p)$ - close to $(\bar{y}, \bar{p})$ - satisfying the required conditions, at least if $y_{0}$ and $y_{1}$ are "small". For a suitable choice of $(\bar{y}, \bar{p})$, we will see that this is indeed true, even if $y_{0}$ and $y_{1}$ are not small. Note that the linearized control system around $(\bar{y}, \bar{p})$ is

$$
\begin{gathered}
\frac{\partial z}{\partial t}-\Delta z+(\bar{y} \cdot \nabla) z+(z \cdot \nabla) \bar{y}+\nabla \pi=0 \text { in }\left(\bar{\Omega} \backslash \Omega^{\#}\right) \times[0, T] \\
\operatorname{div} z=0 \text { in } \bar{\Omega} \times[0, T] \\
z \cdot n=0 \text { on }\left(\Gamma \backslash \Gamma^{\#}\right) \times[0, T], \\
\sigma z \cdot \tau+\operatorname{curl} z=0 \text { on }\left(\Gamma \backslash \Gamma^{\#}\right) \times[0, T] .
\end{gathered}
$$

In [19] J.-L. Lions has proved that if $\bar{y}=0$ this linear system is approximatively controllable; in fact he has treated the no-slip case - i.e. the case where one replaces $(1.28)-(1.29)$ by $z=0$ on $\left(\Gamma \backslash \Gamma^{\#}\right) \times[0, T]$ - but his proof can be easily adapted to the boundary conditions considered here. Unfortunately, if one takes $\bar{y}=0$, it is not clear how to deduce from the approximate controllability of the linear system the existence of $(y, p)$ satisfying (1.12) to (1.17), even if $y_{0}$ and $y_{1}$ are small, for example in a sense given by a $C^{m}$-norm. For this reason, we will not use $(\bar{y}, \bar{p})=(0,0)$, but a $(\bar{y}, \bar{p})$ similar to the one that we have constructed in [8] to prove the controllability of the 2-D Euler equations of incompressible perfect fluids; this $(\bar{y}, \bar{p})$ is in fact "large" so that, in some sense, " $\Delta$ " is small compared to " $(\bar{y} \cdot \nabla)+(\cdot \nabla) \bar{y}$ ".

Our paper is organized as follows: in Section 2 we prove Theorem 1.1 when $\Gamma^{\#}=\emptyset$ and then, in Section 3, deduce the general case from this particular case.

\section{Proof of Theorem 1.1 When $\Gamma^{\#}=\emptyset$}

In this section, we assume that $\Gamma^{\#}=\emptyset$; hence, by (1.1), $\Omega^{\#} \neq \emptyset$.

Let us prove a slightly stronger result than Theorem 1.1; this will be useful when we will study the case $\Gamma^{\#} \neq \emptyset$. Let $\zeta \in C^{\infty}(\Gamma ; \mathbb{R})$ be such that

$$
\int_{\Gamma} \zeta=0
$$

We are going to prove that Theorem 1.1 still holds if, in the statement of this theorem, one replaces (1.8), (1.9) and (1.14) respectively by

$$
\begin{gathered}
y_{0} \cdot n=\zeta \text { on } \Gamma \backslash \Gamma^{\#}, \\
y_{1} \cdot n=\zeta \text { on } \Gamma \backslash \Gamma^{\#}, \\
y(x, t) \cdot n(x)=\zeta(x), \forall(x, t) \in\left(\Gamma \backslash \Gamma^{\#}\right) \times[0, T] .
\end{gathered}
$$

Note that Theorem 1.1 corresponds to the case $\zeta=0$ and that $\zeta$ is given (it is not a control). 
Of course, reducing if necessary $\Omega^{\#}$, we may assume without loss of generality that

$$
\begin{aligned}
& \overline{\Omega^{\#}} \subset \Omega, \\
& \bar{\circ} \\
& \overline{\Omega^{\#}}=\Omega^{\#} .
\end{aligned}
$$

Let us assume the following proposition, whose proof is given in Appendix A,

Proposition 2.1. There exists a constant $C^{*}$ and there exists a function $\bar{C}:(0,+\infty) \rightarrow(0,+\infty)$, such that, for all $\varepsilon>0$, there exist $\theta^{\varepsilon} \in C^{\infty}(\bar{\Omega} \times$ $[0, T] ; \mathbb{R})$ and $\bar{y}^{\varepsilon} \in C^{\infty}\left(\bar{\Omega} \times[0, T] ; \mathbb{R}^{2}\right)$ satisfying

$$
\begin{gathered}
\Delta \theta^{\varepsilon}=0 \text { in }\left(\bar{\Omega} \backslash \Omega^{\#}\right) \times[0, T], \\
\frac{\partial \theta^{\varepsilon}}{\partial n}=0 \text { on } \Gamma \times[0, T],
\end{gathered}
$$

Support $\theta^{\varepsilon}$ is included in $\bar{\Omega} \times[T / 4, T)$,

Support $\bar{y}^{\varepsilon}$ is included in $\bar{\Omega} \times[T / 4, T)$,

$$
\begin{gathered}
\left|\frac{\partial \theta^{\varepsilon}}{\partial \tau}\right|+\left|\frac{\partial^{2} \theta^{\varepsilon}}{\partial \tau^{2}}\right| \leqslant \varepsilon \text { on } \Gamma \times[0, T], \\
\bar{y}^{\varepsilon}=\nabla \theta^{\varepsilon} \text { in }\left(\bar{\Omega} \backslash \Omega^{\#}\right) \times[0, T],
\end{gathered}
$$

$$
\operatorname{div} \bar{y}^{\varepsilon}=0 \text { in } \bar{\Omega} \times[0, T] \text {, }
$$

and such that the following property holds: for all $\left(z_{0}, z_{1}\right)$ in $C^{\infty}\left(\bar{\Omega} ; \mathbb{R}^{2}\right)^{2}$ such that

$$
\begin{aligned}
& \text { div } z_{0}=0 \text { in } \bar{\Omega}, \\
& \text { div } z_{1}=0 \text { in } \bar{\Omega}, \\
& z_{0} \cdot n=\zeta \text { on } \Gamma, \\
& z_{1} \cdot n=\zeta \text { on } \Gamma,
\end{aligned}
$$

there exist $z^{\varepsilon}=Z^{\varepsilon}\left(z_{0}, z_{1}\right)$ in $C^{\infty}\left(\bar{\Omega} \times[0, T] ; \mathbb{R}^{2}\right)$ and $\pi^{\varepsilon}=\Pi^{\varepsilon}\left(z_{0}, z_{1}\right)$ in $C^{\infty}(\bar{\Omega} \times[0, T] ; \mathbb{R})$ satisfying

$$
\begin{gathered}
\frac{\partial z^{\varepsilon}}{\partial t}+\left(\bar{y}^{\varepsilon} \cdot \nabla\right) z^{\varepsilon}+\left(z^{\varepsilon} \cdot \nabla\right) \bar{y}^{\varepsilon}+\nabla \pi^{\varepsilon}=0 \text { in }\left(\bar{\Omega} \backslash \Omega^{\#}\right) \times[0, T], \\
\pi^{\varepsilon}=0 \text { in } \bar{\Omega} \times[0, T / 4], \\
z^{\varepsilon}=z_{0} \text { in } \bar{\Omega} \times[0, T / 4], \\
z^{\varepsilon}(x, T)=z_{1}(x), \forall x \in \Omega \text { such that } d(x) \geqslant \varepsilon, \\
\operatorname{div} z^{\varepsilon}=0 \text { in } \bar{\Omega} \times[0, T], \\
z^{\varepsilon}(x, t) \cdot n(x)=\zeta(x), \forall(x, t) \in \Gamma \times[0, T],
\end{gathered}
$$




$$
\begin{aligned}
&\left|z^{\varepsilon}(\cdot, T)\right|_{L^{\infty}(\Omega)} \leqslant C^{*}\left(\left|z_{0}\right|_{L^{2}(\Omega)}\right.+\left|z_{1}\right|_{L^{2}(\Omega)} \\
&\left.+\left|\operatorname{curl} z_{0}\right|_{L^{\infty}(\Omega)}+\mid \text { curl }\left.z_{1}\right|_{L^{\infty}(\Omega)}\right) \\
&\left|z^{\varepsilon}\right|_{C^{3}\left(\bar{\Omega} \times[0, T] ; \mathbb{R}^{2}\right)} \leqslant \bar{C}(\varepsilon)\left(\left|z_{0}\right|_{C^{4}\left(\bar{\Omega} ; \mathbb{R}^{2}\right)}+\left|z_{1}\right|_{C^{4}\left(\bar{\Omega} ; \mathbb{R}^{2}\right)}\right) .
\end{aligned}
$$

Before going into the details of the proof, let us first briefly explain how we use Proposition 2.1 to construct solutions $(y, p)$ to our controllability problem. We choose $\delta>0$ "very small". During the interval of time [0, (1$\delta) T]$ we use no control: on this interval of time $(y, p)$ is a solution of (1.12), (1.13), (1.15) (1.16), and (2.4) with $\Omega^{\#}=\emptyset$ and $\Gamma^{\#}=\emptyset$. During the interval of time $[(1-\delta) T, T]$ we decompose $(y, p)$ in the following way

$$
y=\bar{y}+z+R, p=\bar{p}+\pi+q,
$$

where

- the map $\bar{y}$ is obtained by the following scaling of $\bar{y}^{\varepsilon}$

$$
\bar{y}(x, t)=\frac{1}{\delta} \bar{y}^{\varepsilon}\left(x, \frac{t-(1-\delta) T}{\delta}\right)
$$

and the function $\bar{p}$ is defined by

$$
\bar{p}(x, t)=-\frac{1}{\delta^{2}}\left(\frac{\partial \theta^{\varepsilon}}{\partial t}+\frac{1}{2}\left|\nabla \theta^{\varepsilon}\right|^{2}\right)\left(x, \frac{t-(1-\delta) T}{\delta}\right),
$$

where $\bar{y}^{\varepsilon}$ and $\theta^{\varepsilon}$ are defined in Proposition 2.1. Let us emphasize that $(\bar{y}, \bar{p})$ satisfies $(1.12),(1.13)$, and (1.14); moreover it satisfies "almost" (1.15), at least if $\varepsilon$ is small enough (see in particular (2.11)).

- the functions $z$ and $\pi$ are obtained by scaling $z^{\varepsilon}$ and $\pi^{\varepsilon}$ in the following way

$$
\begin{gathered}
z(x, t)=z^{\varepsilon}\left(x, \frac{t-(1-\delta) T}{\delta}\right), \\
\pi(x, t)=\frac{1}{\delta} \pi^{\varepsilon}\left(x, \frac{t-(1-\delta) T}{\delta}\right),
\end{gathered}
$$

where $z^{\varepsilon}$ and $\pi^{\varepsilon}$ are defined in Proposition 2.1 by taking

$$
z_{0}=y(\cdot,(1-\delta) T), z_{1}=y_{1} .
$$

- $(R, q)$ is a correction term defined so that $(y, p)$ is a solution of $(1.12)$, $(1.13),(1.15)$, and $(2.4)$, with $[(1-\delta) T, T]$ instead of $[0, T]$.

Note that, by construction,

$$
y(\cdot, T)=z^{\varepsilon}(x, T)+R
$$

and $z^{\varepsilon}(\cdot, T)$ is "close" to $y_{1}$ if $\varepsilon$ is "small". So it suffices to check that $R$ is "small". We will prove that this is indeed the case if " $\varepsilon$ is small and $\delta$ is very small". Rouhgly speaking the reasons are the following. For $\delta$ "very small" the first leading term of

$$
\frac{\partial(\bar{y}+z)}{\partial t}-\Delta(\bar{y}+z)+((\bar{y}+z) \cdot \nabla)(\bar{y}+z)+\nabla(\bar{p}+\pi)
$$

is the term of order $1 / \delta^{2}$, which is

$$
\frac{\partial \bar{y}}{\partial t}+(\bar{y} \cdot \nabla) \bar{y}+\nabla \bar{p}
$$


and the second leading term is the term of order $1 / \delta$, which is

$$
\frac{\partial z}{\partial t}-\Delta \bar{y}+(\bar{y} \cdot \nabla) z+(z \cdot \nabla) \bar{y}+\nabla \pi
$$

By construction these two terms vanish. Moreover $\bar{y}+z$ satisfies (2.4) and, for $\delta$ "small", the leading term of $\bar{y}+z$, which is $\bar{y}$, satifies "almost" (1.15) if $\varepsilon$ is "small". Note that, in the case where $\sigma=0$ on $\Gamma$, $\bar{y}$ satifies (1.15) exactly for all $\varepsilon$. This is why, as mentioned above in b) of Remark 1.2 , we get a better convergence in this case; see also a) of Remark 2.4 below.

Let us now give the details of the proof. Let $y^{*}$ in $C^{\infty}\left(\bar{\Omega} \times[0, T] ; \mathbb{R}^{2}\right)$ and $p^{*}$ in $C^{\infty}(\bar{\Omega} \times[0, T] ; \mathbb{R})$ be such that

$$
\begin{gathered}
\frac{\partial y^{*}}{\partial t}-\Delta y^{*}+\left(y^{*} \cdot \nabla\right) y^{*}+\nabla p^{*}=0 \text { in } \bar{\Omega} \times[0, T], \\
\operatorname{div} y^{*}=0 \text { in } \bar{\Omega} \times[0, T], \\
y^{*} \cdot n=\zeta \text { on } \Gamma \times[0, T], \\
\sigma y^{*} \cdot \tau+\operatorname{curl} y^{*}=0 \text { on } \Gamma \times[0, T], \\
y^{*}(\cdot, 0)=y_{0} \text { in } \bar{\Omega} .
\end{gathered}
$$

The existence (and uniqueness up to an arbitrary function depending only on time added to $p^{*}$ ) can be proved by standard techniques; see [18, Chapitre 1 , Théorème 6.10$]$ for the case $\sigma=0, \zeta=0$, and $\Omega$ simply connected; see also [23] for 3-D Navier-Stokes equations.

Let $\delta \in(0,1 / 2]$ and let $Q^{\delta}=\bar{\Omega} \times[(1-\delta) T, T]$. For $\varepsilon>0$, let $\bar{y}^{\varepsilon, \delta} \in$ $C^{\infty}\left(Q^{\delta} ; \mathbb{R}^{2}\right), z^{\varepsilon, \delta} \in C^{\infty}\left(Q^{\delta} ; \mathbb{R}^{2}\right), \bar{p}^{\varepsilon, \delta} \in C^{\infty}\left(Q^{\delta} ; \mathbb{R}\right), \pi^{\varepsilon, \delta} \in C^{\infty}\left(Q^{\delta} ; \mathbb{R}\right)$ be defined by

$$
\begin{gathered}
\bar{y}^{\varepsilon, \delta}(x, t)=\frac{1}{\delta} \bar{y}^{\varepsilon}\left(x, \frac{t-(1-\delta) T}{\delta}\right) \\
z^{\varepsilon, \delta}(x, t)=Z^{\varepsilon}\left(y^{*}(\cdot,(1-\delta) T), y_{1}\right)\left(x, \frac{t-(1-\delta) T}{\delta}\right), \\
\bar{p}^{\varepsilon, \delta}(x, t)=-\frac{1}{\delta^{2}}\left(\frac{\partial \theta^{\varepsilon}}{\partial t}+\frac{1}{2}\left|\nabla \theta^{\varepsilon}\right|^{2}\right)\left(x, \frac{t-(1-\delta) T}{\delta}\right), \\
\pi^{\varepsilon, \delta}(x, t)=\frac{1}{\delta} \Pi^{\varepsilon}\left(y^{*}(\cdot,(1-\delta) T), y_{1}\right)\left(x, \frac{t-(1-\delta) T}{\delta}\right),
\end{gathered}
$$

for all $(x, t) \in Q^{\delta}$. Note that, by (2.10) and (2.31),

$$
\bar{y}^{\varepsilon, \delta}=0 \text { in } \bar{\Omega} \times[(1-\delta) T, T-(3 \delta / 4) T],
$$

and, by (2.20) and (2.32),

$$
z^{\varepsilon, \delta}(x, t)=y^{*}(x,(1-\delta) T), \forall(x, t) \in \bar{\Omega} \times[(1-\delta) T, T-(3 \delta / 4) T] .
$$

Similarly, by (2.9) and (2.33),

$$
\bar{p}^{\varepsilon, \delta}=0 \text { in } \bar{\Omega} \times[(1-\delta) T, T-(3 \delta / 4) T]
$$

and, by (2.19) and (2.34),

$$
\pi^{\varepsilon, \delta}=0 \text { in } \bar{\Omega} \times[(1-\delta) T, T-(3 \delta / 4) T] .
$$


Let $F^{\varepsilon, \delta}: \bar{\Omega} \times[0, T] \rightarrow \mathbb{R}^{2}$ be defined by

$$
\begin{gathered}
F^{\varepsilon, \delta}=0 \text { in } \bar{\Omega} \times[0,(1-\delta) T), \\
F^{\varepsilon, \delta}=\frac{\partial}{\partial t}\left(\bar{y}^{\varepsilon, \delta}+z^{\varepsilon, \delta}\right)-\Delta \bar{y}^{\varepsilon, \delta}+\left(\bar{y}^{\varepsilon, \delta} \cdot \nabla\right) z^{\varepsilon, \delta}+\left(z^{\varepsilon, \delta} \cdot \nabla\right) \bar{y}^{\varepsilon, \delta} \\
+\left(\bar{y}^{\varepsilon, \delta} \cdot \nabla\right) \bar{y}^{\varepsilon, \delta}+\nabla \bar{p}^{\varepsilon, \delta}+\nabla \pi^{\varepsilon, \delta} \text { in } Q^{\delta} .
\end{gathered}
$$

Then - see in particular $(2.35)$ to $(2.40)-F^{\varepsilon, \delta} \in C^{\infty}\left(\bar{\Omega} \times[0, T] ; \mathbb{R}^{2}\right)$. For $a=\left(a_{1}, a_{2}\right) \in \mathbb{R}^{2}$, let $a^{\perp}=\left(-a_{2}, a_{1}\right)$. By standard techniques - see also (2.35) -, one shows the existence of $y^{\varepsilon, \delta}$ in $C^{\infty}\left(\bar{\Omega} \times[0, T] ; \mathbb{R}^{2}\right)$ and $p^{\varepsilon, \delta}$ in $C^{\infty}(\bar{\Omega} \times[0, T] ; \mathbb{R})$ such that

$$
\begin{gathered}
\frac{\partial y^{\varepsilon, \delta}}{\partial t}-\Delta y^{\varepsilon, \delta}+\left(y^{\varepsilon, \delta} \cdot \nabla\right) y^{\varepsilon, \delta}+\nabla p^{\varepsilon, \delta}=0 \text { in } \bar{\Omega} \times[0,(1-\delta) T], \\
\frac{\partial y^{\varepsilon, \delta}}{\partial t}-\Delta y^{\varepsilon, \delta}+\left(y^{\varepsilon, \delta} \cdot \nabla\right) y^{\varepsilon, \delta}+\nabla p^{\varepsilon, \delta}=F^{\varepsilon, \delta} \\
+\left(\operatorname{curl} \bar{y}^{\varepsilon, \delta}\right)\left(y^{\varepsilon, \delta}-\bar{y}^{\varepsilon, \delta}-z^{\varepsilon, \delta}\right)^{\perp} \text { in } \bar{\Omega} \times[(1-\delta) T, T], \\
\operatorname{div} y^{\varepsilon, \delta}=0 \text { in } \bar{\Omega} \times[0, T], \\
y^{\varepsilon, \delta} \cdot n=\zeta \text { on } \Gamma \times[0, T], \\
\sigma y^{\varepsilon, \delta} \cdot \tau+\operatorname{curl} y^{\varepsilon, \delta}=0 \text { on } \Gamma \times[0, T], \\
y^{\varepsilon, \delta}(\cdot, 0)=y_{0} \text { in } \bar{\Omega} .
\end{gathered}
$$

For simplicity, let us write $y, p, z, \pi, \bar{y}, \bar{p}, F, Q$ instead of $y^{\varepsilon, \delta}, p^{\varepsilon, \delta}, z^{\varepsilon, \delta}, \pi^{\varepsilon, \delta}$, $\bar{y}^{\varepsilon, \delta}, \bar{p}^{\varepsilon, \delta}, F^{\varepsilon, \delta}, Q^{\delta}$. From (2.7), (2.12), (2.6), (2.31), and (2.33), we have

$$
\begin{gathered}
\frac{\partial \bar{y}}{\partial t}-\Delta \bar{y}+\bar{y} \cdot \nabla \bar{y}+\nabla \bar{p}=0 \text { in }\left(\bar{\Omega} \backslash \Omega^{\#}\right) \times[(1-\delta) T, T], \\
\operatorname{curl} \bar{y}=0 \text { in }\left(\bar{\Omega} \backslash \Omega^{\#}\right) \times[(1-\delta) T, T] .
\end{gathered}
$$

From (2.18), (2.31), (2.32), and (2.34), we get

$$
\frac{\partial z}{\partial t}+(\bar{y} \cdot \nabla) z+(z \cdot \nabla) \bar{y}+\nabla \pi=0 \text { in }\left(\bar{\Omega} \backslash \Omega^{\#}\right) \times[(1-\delta) T, T],
$$

which, with (2.40) and (2.47), implies that

$$
F=0 \text { in }\left(\bar{\Omega} \backslash \Omega^{\#}\right) \times[(1-\delta) T, T] .
$$

From (2.41), (2.42), (2.48), and (2.49), we obtain (1.12). From (2.43) to $(2.46)$, we obtain $(1.13),(1.15),(1.16)$, and $(2.4)$. So, in order to finish the proof, it remains only to check that, given a compact $K \subset \Omega$, given $\mu>0$ and $\nu>0$, we have, for a suitable choice of $\varepsilon$ and $\delta$,

$$
\begin{gathered}
\int_{\Omega} d^{\mu}\left|y(\cdot, T)-y_{1}\right|<\nu, \\
\left|y(\cdot, T)-y_{1}\right|_{W-1, \infty(\Omega)}<\nu,
\end{gathered}
$$

$\left|\operatorname{curl} y(\cdot, T)-\operatorname{curl} y_{1}\right|_{L^{\infty}(K)}<\nu$. 
Let us first point out that, by (2.26) to (2.30), (2.41), and (2.43) to (2.46), we have

$$
y=y^{*} \text { on } \bar{\Omega} \times[0,(1-\delta) T] .
$$

Let $R \in C^{\infty}\left(Q ; \mathbb{R}^{2}\right)$ and $q \in C^{\infty}(Q ; \mathbb{R})$ be defined by

$$
\begin{aligned}
& R=y-\bar{y}-z \\
& q=p-\bar{p}-\pi .
\end{aligned}
$$

By $(2.40),(2.42),(2.54)$, and $(2.55)$, we have

$$
\begin{aligned}
\frac{\partial R}{\partial t}-\Delta R+((R+\bar{y}+z) \cdot \nabla) R+(R \cdot \nabla)(\bar{y}+z) \\
-\Delta z+(z \cdot \nabla) z-(\operatorname{curl} \bar{y}) R^{\perp}+\nabla q=0 \text { in } Q .
\end{aligned}
$$

From $(2.13),(2.22),(2.31),(2.32),(2.43)$, and (2.54), we get

$$
\operatorname{div} R=0 \text { in } Q \text {. }
$$

From $(2.8),(2.12),(2.23),(2.31),(2.32),(2.44)$, and (2.54), we obtain, with $I=[(1-\delta) T, T]$,

$$
R \cdot n=0 \text { on } \Gamma \times I \text {. }
$$

From $(2.7),(2.12),(2.5),(2.31),(2.32),(2.45)$, and (2.54), we get, with $\omega=\operatorname{curl} R$,

$$
\sigma R \cdot \tau+\omega=-\sigma \bar{y} \cdot \tau-\sigma z \cdot \tau-\operatorname{curl} z \text { on } \Gamma \times I .
$$

From $(2.10),(2.31)$, and (2.54), we get

$$
R=y-z \text { on } \bar{\Omega} \times\{T\} .
$$

We fix a compact $K \subset \Omega$ and two real numbers $\mu>0$ and $\nu>0$. By (2.21), (2.24), and (2.32), there exists $\varepsilon_{0}>0$ such that, for any $\varepsilon \in\left(0, \varepsilon_{0}\right]$ and for any $\delta \in(0,1 / 2]$,

$$
\begin{gathered}
\int_{\Omega} d^{\mu}\left|z(\cdot, T)-y_{1}\right|<\nu / 2, \\
\left|z(\cdot, T)-y_{1}\right|_{W-1, \infty(\Omega)}<\nu / 2, \\
\operatorname{curl} z(\cdot, T)=\text { curl } y_{1} \text { on } K .
\end{gathered}
$$

Hence, by (2.60), in order to get $(2.50),(2.51)$, and (2.52), it suffices to check that, for suitable choices of $\varepsilon \in\left(0, \varepsilon_{0}\right]$ and of $\delta \in(0,1 / 2]$,

$$
\begin{gathered}
\int_{\Omega} d^{\mu}|R(\cdot, T)|<\nu / 2, \\
|R(\cdot, T)|_{W-1, \infty(\Omega)}<\nu / 2, \\
|\omega(\cdot, T)|_{L^{\infty}(K)}<\nu .
\end{gathered}
$$

From $(2.10),(2.20),(2.31),(2.32),(2.53)$, and (2.54), we have

$$
R=0 \text { on } \bar{\Omega} \times\{(1-\delta) T\} .
$$

Let us denote by $C_{j}(\varepsilon), j \geqslant 1$, various positive constants which may depend on $T, y_{0}, y_{1}, \ldots, \Omega^{\#}$, and $\varepsilon$, but are independent of $\delta$ in $(0,1 / 2]$ and of $s$ in $I$. Furthermore let us denote by $C_{j}, j \geqslant 1$, various positive constants which Esaim: CoCV, May 1996, VOL. 1, PP. 35-75. 
may depend on $T, y_{0}, y_{1}, \ldots, \Omega^{\#}$, but are independent of $\delta$ in $(0,1 / 2]$, of $s$ in $I$, and of $\varepsilon$ in $(0,1]$. For example, from $(2.11),(2.12),(2.25),(2.31),(2.32)$, and (2.59), we get the existence of $C_{1}>0$ and $C_{1}(\varepsilon)>0$ such that, for all $\varepsilon \in(0,1]$ and for all $\delta \in\left(0, C_{1}(\varepsilon)^{-1}\right]$,

$$
|\omega|_{L^{\infty}(\Gamma \times[(1-\delta) T, s]} \leqslant C_{1}\left(\frac{\varepsilon}{\delta}+|R|_{L^{\infty}(\bar{\Omega} \times[(1-\delta) T, s]}\right), \forall s \in I .
$$

Taking the curl of (2.56), we get, using (2.13), (2.22), (2.31), (2.32), and $(2.57)$,

$$
\begin{aligned}
\frac{\partial \omega}{\partial t}-\Delta \omega+((R+\bar{y}+z) & \cdot \nabla) \omega+(R \cdot \nabla) \operatorname{curl} z \\
& -\Delta(\operatorname{curl} z)+(z \cdot \nabla)(\operatorname{curl} z)=0 \text { in } Q .
\end{aligned}
$$

For $s \in I$, let

$$
I^{\prime}=[(1-\delta) T, s], Q^{\prime}=\bar{\Omega} \times I^{\prime} .
$$

In order to obtain a pointwise estimate on $\omega$ one uses the following lemma, whose proof is given in Appendix B,

Lemma 2.2. Let $f \in C^{\infty}([0,+\infty) ;[0,+\infty))$ be such that

$$
\begin{gathered}
f(s)=s, \forall s \in[0,1], \\
0 \leqslant f^{\prime} \leqslant 1 \text { in }[0,+\infty), \\
f=\frac{3}{2} \text { in }[2,+\infty) .
\end{gathered}
$$

Let $\alpha \in(0,1)$ and $\beta \in(0,1)$ be such that

$$
\alpha+\beta=1 \text {. }
$$

Then there exists a positive real number $C$ such that, for any $t^{*} \in\left(0, C^{-1}\right]$, for any $\psi \in C^{\infty}\left(\bar{\Omega} \times\left[0, t^{*}\right] ; \mathbb{R}\right)$, for any $X \in C^{\infty}\left(\bar{\Omega} \times\left[0, t^{*}\right] ; \mathbb{R}^{2}\right)$, and for any $Y \in C^{\infty}\left(\bar{\Omega} \times\left[0, t^{*}\right] ; \mathbb{R}^{2}\right)$ such that

$$
\begin{gathered}
\frac{\partial \psi}{\partial t}-\Delta \psi+((X+Y) \cdot \nabla) \psi \leqslant 0 \text { in } \bar{\Omega} \times\left[0, t^{*}\right], \\
\psi(\cdot, 0) \leqslant 0 \text { in } \bar{\Omega} \\
\psi \leqslant 1 \text { on } \Gamma \times\left[0, t^{*}\right] \\
\left|\frac{\partial Y}{\partial n}\right| \leqslant C^{-1} t^{*-1} \text { on } \Gamma \times\left[0, t^{*}\right] \\
Y \cdot n=0 \text { on } \Gamma \times\left[0, t^{*}\right]
\end{gathered}
$$

we have, for all $x$ in $\bar{\Omega}$ and all $t$ in $\left(0, t^{*}\right]$,

$$
\begin{aligned}
\psi(x, t) \leqslant\left(\exp C t|X|_{L^{\infty}\left(\Omega \times\left[0, t^{*}\right]\right)}^{2}\right)( & \left.\exp C t^{\frac{3 \beta}{2}}\left|\nabla^{2} Y\right|_{L^{\infty}\left(\Omega \times\left[0, t^{*}\right]\right)}\right) \\
& \left(\exp -\left(\frac{1}{(C t)^{\alpha}} f\left(\frac{d^{2}}{(C t)^{\beta}}\right)\right)\right) .
\end{aligned}
$$


In (2.76) as well as in the remaining part of this paper

$$
\begin{aligned}
\left|\nabla^{2} Y\right|_{L^{\infty}\left(\Omega \times\left[0, t^{*}\right]\right)}=\left|\frac{\partial^{2} Y}{\partial x_{1}^{2}}\right|_{L^{\infty}\left(\Omega \times\left[0, t^{*}\right]\right)} & \\
& +\left|\frac{\partial^{2} Y}{\partial x_{1} \partial x_{2}}\right|_{L_{\left(\Omega \times\left[0, t^{*}\right]\right)}}+\left|\frac{\partial^{2} Y}{\partial x_{2}^{2}}\right|_{L^{\infty}\left(\Omega \times\left[0, t^{*}\right]\right)}
\end{aligned}
$$

We take $\delta \in\left(0, C_{1}(\varepsilon)^{-1}\right]$ and we apply this lemma with

$$
\begin{gathered}
X(x, t)=(R+z)(x, t+(1-\delta) T), \\
Y(x, t)=\bar{y}(x, t+(1-\delta) T), \\
\psi(x, t)=\frac{1}{C_{1}\left(\frac{\varepsilon}{\delta}+|R|_{L^{\infty}\left(Q^{\prime}\right)}\right)}[ \pm \omega(x, t+(1-\delta) T) \\
\left.-t|R|_{L^{\infty}\left(Q^{\prime}\right)}|\nabla \operatorname{curl} z|_{L^{\infty}\left(Q^{\prime}\right)}-t|-\Delta \operatorname{curl} z+(z \cdot \nabla) \operatorname{curl} z|_{L^{\infty}\left(Q^{\prime}\right)}\right], \\
\alpha=\frac{1}{4}, \\
\beta=\frac{3}{4}, \\
t^{*}=s-(1-\delta) T(\leqslant \delta T) .
\end{gathered}
$$

Using (2.66), and (2.77) to (2.79), we get (2.71). From (2.64) and (2.79), we get (2.72). From (2.65) and (2.79), we get (2.73). Note that, by (2.7), (2.11), (2.12), (2.31), and (2.78), (2.74) holds if, for some $C_{2}>0, \varepsilon<C_{2}^{-1}$ and for some $C_{2}(\varepsilon)>0, \delta \in\left(0, C_{2}(\varepsilon)^{-1}\right]$. From (2.8), (2.12), (2.31), and (2.78), we get (2.75). Moreover, by (2.31), (2.78), (2.81), and (2.82), we have, for some $C_{3}(\varepsilon)>0$,

$$
t^{3 \beta / 2}\left|\nabla^{2} Y\right|_{L^{\infty}\left(\Omega \times\left[0, t^{*}\right]\right)} \leqslant 1, \forall t \in\left[0, t^{*}\right], \quad \forall \delta \in\left(0, C_{3}(\varepsilon)^{-1}\right] .
$$

We choose a function $f \in C^{\infty}([0,+\infty) ;[0,+\infty))$ satisfying $(2.67)$ to $(2.69)$ and apply Lemma 2.2 together with $(2.25),(2.32),(2.77)$, and (2.83); we get the existence of $C_{4}>0$ and $C_{4}(\varepsilon)>0$ such that, if $\varepsilon \in\left(0, C_{4}^{-1}\right]$, if $\delta \in\left(0, C_{4}(\varepsilon)^{-1}\right]$, and if

$$
\delta|R|_{L^{\infty}\left(Q^{\prime}\right)}^{2} \leqslant 1
$$

then, for all $(x, t)$ in $\bar{\Omega} \times[(1-\delta) T, s]$,

$$
\begin{aligned}
|\omega(x, t)| \leqslant C_{4}(\varepsilon)\left(\delta|R|_{L^{\infty}\left(Q^{\prime}\right)}+\delta\right) & \\
& +C_{4}\left(\frac{\varepsilon}{\delta}+|R|_{L^{\infty}\left(Q^{\prime}\right)}\right) \exp \left(-\frac{1}{\left(C_{4} \delta\right)^{\alpha}} f\left(\frac{d^{2}(x)}{\left(C_{4} \delta\right)^{\beta}}\right)\right) .
\end{aligned}
$$

In order to deduce estimates on $R$ from (2.85), we use the following lemma, whose proof is given in Appendix $C$,

Esaim: CoCv, May 1996, Vol. 1, PP. 35-75. 
Lemma 2.3. Let $f \in C^{\infty}([0,+\infty) ;[0,+\infty))$ be such that

$$
\begin{gathered}
f(s)=s, \forall s \in[0,1], \\
1 \leqslant f(s) \leqslant s, \forall s \in[1,+\infty) .
\end{gathered}
$$

Let $\alpha \in(0,1]$ and let $\beta \in[0,1)$ be such that (2.70) holds. Then there exists a positive real number $C$ such that, for any $A$ in $[1,+\infty)$, for any $B$ in $[0,+\infty)$, for any $B^{*}$ in $[0,+\infty)$, and for any $\psi$ in $C^{1}(\bar{\Omega} ; \mathbb{R})$ such that $\Delta \psi \in L^{\infty}(\Omega)$ and satisfying

$$
\begin{gathered}
|\Delta \psi| \leqslant B+B^{\star} \exp \left(-A^{\alpha} f\left(A^{\beta} d^{2}\right)\right) \text { in } \Omega \\
\psi=0 \text { on } \Gamma
\end{gathered}
$$

one has

$$
\begin{gathered}
|\nabla \psi| \leqslant C\left(B+\frac{B^{*}}{\sqrt{A}}\right) \text { in } \bar{\Omega} \\
\left|d^{\mu} \nabla \psi\right|_{L^{1}(\Omega)} \leqslant C\left(B+\frac{B^{*}}{\mu A}\right), \forall \mu \in(0,1], \\
|\psi| \leqslant C\left(B+\frac{B^{*}}{A}\right) \text { in } \bar{\Omega} .
\end{gathered}
$$

Let us first finish the proof of Theorem 1.1 when $\Omega$ is simply connected (with $\Gamma^{\#}=\emptyset$, and with $(2.2),(2.3)$, and (2.4) instead of (1.8), (1.9), and (1.14) ). Then one can write

$$
R=\nabla^{\perp} \varphi:=(\nabla \varphi)^{\perp},
$$

where $\varphi \in C^{\infty}(\bar{\Omega} ; \mathbb{R})$ satisfies

$$
\begin{gathered}
\Delta \varphi=\omega \text { in } \bar{\Omega}, \\
\varphi=0 \text { on } \Gamma .
\end{gathered}
$$

Then, it follows from (2.85) and Lemma 2.3 - see (2.90) - that there exist $C_{5}>0$ and $C_{5}(\varepsilon)>C_{4}(\varepsilon)$ such that, if $\varepsilon \in\left(0, C_{5}^{-1}\right)$, if $\delta \in\left(0, C_{5}(\varepsilon)^{-1}\right]$, and if (2.84) holds,

$$
|R|_{L^{\infty}\left(Q^{\prime}\right)} \leqslant C_{5} \frac{\varepsilon}{\sqrt{\delta}} .
$$

Hence, if $\varepsilon \in\left(0, C_{5}^{-1} / 2\right]$ and if $\delta \in\left(0, C_{5}(\varepsilon)^{-1}\right]$, then

$$
(2.84) \Rightarrow\left(\sqrt{\delta}|R|_{L^{\infty}\left(Q^{\prime}\right)} \leqslant C_{5} \varepsilon \leqslant \frac{1}{2}\right) .
$$

Note that, by $(2.64),(2.84)$ holds if $s$ is close enough to $(1-\delta) T$. Hence, by $(2.96),(2.84)$ holds for all $s \in I$ if $\varepsilon \in\left(0, C_{5}^{-1} / 2\right]$ and $\delta \in\left(0, C_{5}(\varepsilon)^{-1}\right]$; therefore, by (2.96),

$$
|R|_{L^{\infty}(Q)} \leqslant C_{5} \frac{\varepsilon}{\sqrt{\delta}}, \forall \varepsilon \in\left(0, C_{5}^{-1} / 2\right], \forall \delta \in\left(0, C_{5}(\varepsilon)^{-1}\right] .
$$

Using (2.85), (2.97), and Lemma 2.3 - more precisely (2.91) and (2.92) -, we have shown the existence of $C_{6}>0$ and $C_{6}(\varepsilon)>C_{5}(\varepsilon)$ such that, for any $\varepsilon$ in $\left(0, C_{6}^{-1}\right]$, any $\delta$ in $\left(0, C_{6}(\varepsilon)^{-1}\right]$, and any $\mu$ in $(0,1]$,

$$
|\omega| \leqslant C_{6} \varepsilon \text { in } K \times[(1-\delta) T, T],
$$




$$
\begin{aligned}
& |\varphi| \leqslant C_{6} \varepsilon \text { in } \bar{\Omega} \times[(1-\delta) T, T], \\
& \left|d^{\mu} \nabla \varphi(\cdot, T)\right|_{L^{1}(\Omega)} \leqslant \frac{C_{6}}{\mu} \varepsilon .
\end{aligned}
$$

These imply (2.61) to (2.63) if, for some $C_{7}>0$ and $C_{7}(\varepsilon)>0, \varepsilon \in\left(0, C_{7}^{-1}\right]$ and $\delta \in\left(0, C_{7}(\varepsilon)^{-1}\right]$.

We now turn to the case where $\Omega$ is not simply connected. Let $\Gamma_{0}, \ldots, \Gamma_{i}$, $\ldots, \Gamma_{g}$ be the connected components of $\Gamma$. For $i \in[1, g]$, let $\xi^{i} \in C^{\infty}(\bar{\Omega} ; \mathbb{R})$ be defined by

$$
\begin{gathered}
\Delta \xi^{i}=0 \text { in } \bar{\Omega}, \\
\xi^{i}=0 \text { on } \Gamma \backslash \Gamma_{i}, \\
\xi^{i}=1 \text { on } \Gamma_{i} .
\end{gathered}
$$

Let $\varphi \in C^{\infty}(\bar{\Omega} ; \mathbb{R})$ be still defined by $(2.94)$ and $(2.95)$. Then there exists a (unique) $\lambda=\left(\lambda_{1}, \ldots, \lambda_{g}\right) \in \mathbb{R}^{g}$ such that

$$
R=\nabla^{\perp} \varphi+\sum_{i=1}^{g} \lambda_{i} \nabla^{\perp} \xi^{i} .
$$

With suitable integration by parts, one easily shows, using $(2.23),(2.32)$, (2.94), (2.95), (2.99), (2.100), (2.101), and (2.102), that, for all $i \in[1, g]$,

$$
\begin{gathered}
\int_{\Omega} \frac{\partial R}{\partial t} \cdot \nabla^{\perp} \xi^{i}=\sum_{j=1}^{g}\left(\frac{d \lambda_{j}}{d t} \int_{\Omega} \nabla \xi^{i} \cdot \nabla \xi^{j}\right) \\
\int_{\Omega} \Delta R \cdot \nabla^{\perp} \xi^{i}=\int_{\Gamma} \omega \frac{\partial \xi^{i}}{\partial n} \\
\int_{\Omega}((R \cdot \nabla) R) \cdot \nabla^{\perp} \xi^{i}=\int_{\Omega} \omega\left(\frac{\partial \varphi}{\partial x_{1}} \frac{\partial \xi^{i}}{\partial x_{2}}-\frac{\partial \varphi}{\partial x_{2}} \frac{\partial \xi^{i}}{\partial x_{1}}\right) \\
+\sum_{j=1}^{g} \lambda_{j} \int_{\Omega} \omega\left(\frac{\partial \xi^{j}}{\partial x_{1}} \frac{\partial \xi^{i}}{\partial x_{2}}-\frac{\partial \xi^{j}}{\partial x_{2}} \frac{\partial \xi^{i}}{\partial x_{1}}\right) \\
\int_{\Omega}\left((R \cdot \nabla) \bar{y}+(\bar{y} \cdot \nabla) R-(\mathrm{curl} \bar{y}) R^{\perp}\right) \cdot \nabla^{\perp} \xi^{i}=\int_{\Omega} \omega\left(\bar{y} \cdot \nabla \xi^{i}\right) \\
\int_{\Omega}((R \cdot \nabla) z+(z \cdot \nabla) R) \cdot \nabla^{\perp} \xi^{i}=\int_{\Omega} \mathrm{curl} z \nabla^{\perp} \varphi \cdot \nabla \xi^{i}+\int_{\Gamma} \frac{\partial \varphi}{\partial n} \frac{\partial \xi^{i}}{\partial n} \zeta \\
-\int_{\Omega} \nabla \varphi \cdot \nabla\left(z \cdot \nabla \xi^{i}\right)+\sum_{j=1}^{g} \lambda_{j} \int_{\Gamma_{j}}(\mathrm{curl} z) \nabla^{\perp} \xi^{j} \cdot \nabla \xi^{i} \\
\int_{\Omega} \nabla q \cdot \nabla^{\perp} \xi^{i}=0
\end{gathered}
$$


Let us point out that, by $(2.100)$ and $(2.101)$, one has for any $\left(a_{1}, \ldots ., a_{g}\right)$ in $\mathbb{R}^{g}$,

$$
\left(\sum_{j=1}^{g} a_{j} \nabla^{\perp} \xi^{j}=0\right) \Rightarrow\left(a_{j}=0, \forall j \in[1, g]\right)
$$

Hence

the matrix $\left(\int_{\Omega} \nabla^{\perp} \xi^{i} \cdot \nabla^{\perp} \xi^{j} ; 1 \leqslant i \leqslant g, 1 \leqslant j \leqslant g\right)$ is invertible.(2.109) Let

$$
|\lambda|=\sum_{i=1}^{g}\left|\lambda_{i}\right|
$$

We take the scalar product of (2.56) with $\nabla^{\perp} \xi^{i}$ and integrate the resulting expression over $\Omega$. Then, using (2.25), (2.32), (2.103) to (2.108), and (2.109), one gets that, for some $C_{8}>0$ and $C_{8}(\varepsilon)>0$, one has, for all $i \in[1, g]$,

$$
\begin{aligned}
\left|\frac{d \lambda_{i}}{d t}\right| \leqslant & C_{8}\left(|\omega|_{L^{\infty}\left(Q^{\prime}\right)}+|\omega(\cdot, t)|_{L^{1}(\Omega)}|\nabla \varphi|_{L^{\infty}\left(Q^{\prime}\right)}+|\lambda||\omega(\cdot, t)|_{L^{1}(\Omega)}\right. \\
& \left.+\left|\left(\omega \bar{y} \cdot \nabla \xi^{i}\right)(\cdot, t)\right|_{L^{1}(\Omega)}\right)+C_{8}(\varepsilon)\left(1+|\nabla \varphi|_{L^{\infty}\left(Q^{\prime}\right)}+|\lambda|\right) .
\end{aligned}
$$

Note that, by $(2.5),(2.7),(2.8),(2.11),(2.12),(2.31),(2.100)$, and (2.101), one has, for some $C_{9}>0$ and $C_{9}(\varepsilon)>0$,

$$
\left|\bar{y} \cdot \nabla \xi^{i}\right| \leqslant C_{9} \frac{\varepsilon}{\delta} d+C_{9}(\varepsilon) \frac{d^{2}}{\delta} \text { in } \bar{\Omega}, \forall \varepsilon \in(0,1], \forall \delta \in(0,1] .
$$

Straightforward computations shows that, for some $C_{10}>0$,

$$
\int_{\Omega} d^{l} \exp -A^{\frac{1}{4}} f\left(d^{2} A^{\frac{3}{4}}\right) \leqslant \frac{C_{10}}{A^{\frac{1+l}{2}}}, \quad \forall l \in\{0,1,2\}, \quad \forall A \in[1,+\infty) .
$$

Again, from (2.85) and Lemma 2.3, we get the existence of $C_{11}>0$ and $C_{11}(\varepsilon)>0$ such that, if $\varepsilon \in\left(0, C_{11}^{-1}\right)$, if $\delta \in\left(0, C_{11}(\varepsilon)^{-1}\right]$, and if $(2.84)$ holds,

$$
\begin{gathered}
|\nabla \varphi|_{L^{\infty}\left(Q^{\prime}\right)} \leqslant C_{11} \frac{\varepsilon}{\sqrt{\delta}}, \\
\int_{\Omega}\left|d^{\mu} \nabla \varphi(\cdot, T)\right| \leqslant \frac{C_{11}}{\mu} \varepsilon, \\
|\varphi|_{L^{\infty}\left(Q^{\prime}\right)} \leqslant C_{11} \varepsilon .
\end{gathered}
$$

From (2.85), (2.112), and (2.84), we get the existence of $C_{12}>C_{11}$ and $C_{12}(\varepsilon)>C_{11}(\varepsilon)$ such that, if $\varepsilon \in\left(0, C_{11}^{-1}\right)$, if $\delta \in\left(0, C_{12}(\varepsilon)^{-1}\right]$ and if $(2.84)$ holds, then, for all $t$ in $I$,

$$
\begin{aligned}
&|\omega|_{L^{\infty}\left(Q^{\prime}\right)} \leqslant C_{12} \frac{\varepsilon}{\delta} \\
&|\omega(\cdot, t)|_{L^{1}(\Omega)} \leqslant C_{12} \frac{\varepsilon}{\sqrt{\delta}}, \\
&|d \omega(\cdot, t)|_{L^{1}(\Omega)} \leqslant C_{12} \varepsilon \\
&\left|d^{2} \omega(\cdot, t)\right|_{L^{1}(\Omega)} \leqslant C_{12}(\varepsilon) \sqrt{\delta}, \\
& \text { ESaim: Cocv, MaY 1996, Vol. 1, PP. 35-75. }
\end{aligned}
$$


which, with (2.64), (2.102), (2.110), (2.111), and (2.113), imply that, for some $C_{13}>0$ and some $C_{13}(\varepsilon)>0$, one has, if $\delta \in\left(0, C_{13}(\varepsilon)^{-1}\right]$

$$
|\lambda|_{L^{\infty}\left(I^{\prime}\right)} \leqslant C_{13} \varepsilon \text {. }
$$

In addition, using (2.102), for some $C_{14}>0$, one has

$$
|R|_{L^{\infty}\left(Q^{\prime}\right)} \leqslant C_{14}\left(|\nabla \varphi|_{L^{\infty}\left(Q^{\prime}\right)}+|\lambda|_{L^{\infty}\left(I^{\prime}\right)}\right),
$$

which, with (2.113) and (2.116), implies that

$$
|R|_{L^{\infty}\left(Q^{\prime}\right)} \leqslant C_{14}\left(C_{11} \frac{\varepsilon}{\sqrt{\delta}}+C_{13} \varepsilon\right) .
$$

Hence there exist $C_{15}>0$ and $C_{15}(\varepsilon)>0$ such that, if $\varepsilon \in\left(0, C_{14}^{-1}\right]$ and if $\delta \in\left(0, C_{15}(\varepsilon)^{-1}\right]$, then

$$
(2.84) \Rightarrow\left(\sqrt{\delta}|R|_{L^{\infty}\left(Q^{\prime}\right)} \leqslant C_{15} \varepsilon \leqslant \frac{1}{2}\right) .
$$

Note that, by $(2.64),(2.84)$ holds if $s$ is close enough to $(1-\delta) T$. Hence, by (2.117), (2.84) holds for $Q=Q^{\prime}$ if $\varepsilon \in\left(0, C_{15}^{-1}\right]$ and $\delta \in\left(0, C_{15}(\varepsilon)^{-1}\right]$ which, with (2.85), (2.102), (2.114), (2.115), and (2.116), shows that (2.61) to (2.63) hold if $\varepsilon \in\left(0, C_{16}^{-1}\right]$ and $\delta \in\left(0, C_{16}^{-1}(\varepsilon)\right.$ for some $C_{16}>0$ and some $C_{16}(\varepsilon)>0$. This ends the proof of Theorem 1.1 when $\Gamma^{\#}=\emptyset$.

REMARK 2.4. a) It would be interesting to know if more careful estimates could lead to a better convergence than the one given by (1.21) and (1.22). For example one may wonder if, for suitable choices of $\delta$ and $\varepsilon$, one could get, instead of (1.21) and (1.22), the stronger statement

$$
\left|y^{k}(\cdot, T)-y_{1}\right|_{L^{2}(\Omega)} \rightarrow 0 .
$$

In order to get such a convergence it might be interesting to take for $\delta$ a function of $\varepsilon$, with $\varepsilon$ small enough, and to get some new estimates on $\theta^{\varepsilon}$ and $\bar{y}^{\varepsilon}$ constructed in Appendix A. But, in the special case where

$$
\sigma=0 \text { on } \Gamma,
$$

it follows from our construction that Theorem 1.1 still holds even if one requires also (2.118). Indeed in this case (2.59) becomes

$$
\omega=-\operatorname{curl} z \text { on } \Gamma \times I
$$

and therefore $(2.65)$ becomes

$$
|\omega|_{L^{\infty}(\Gamma \times[(1-\delta) T, T]} \leqslant C_{17}(\varepsilon),
$$

for some $C_{17}(\varepsilon)>0$. Using this inequality and the same arguments as above, we now get that

$$
|R|_{L^{\infty}(Q)} \leqslant C_{18}(\varepsilon) \sqrt{\delta}
$$

if $\varepsilon \in\left(0, C_{18}^{-1}\right]$ and $\delta \in\left(0, C_{18}^{-1}(\varepsilon)\right]$ for some $C_{18}>0$ and some $C_{18}(\varepsilon)>$ 0 . Hence, if we let

$$
\begin{gathered}
\varepsilon=1 / k, \delta=1 /\left(C_{18}(1 / k)+k C_{18}(1 / k)^{2}\right), \\
\left(y^{k}, p^{k}\right)=(y, p)\left(=\left(y^{\varepsilon, \delta}, p^{\varepsilon, \delta}\right)\right),
\end{gathered}
$$

we get (1.23) and - see in particular (2.24) -

$$
\left|y^{k}(\cdot, T)\right|_{L^{\infty}(\Omega)} \leqslant C_{19}, \forall k>C_{18},
$$


which, with (1.23), gives (2.118). Let us point out that, when (2.119) holds, our proof can be simplified. Indeed, in this case,

$$
\text { curl } z=0 \text { on } \Gamma \times I
$$

and so, with (2.120),

$$
\omega=0 \text { on } \Gamma \times I .
$$

From $(2.66),(2.94),(2.95),(2.102),(2.110)$, and (2.122), we get, without using Lemma 2.2 and without using Lemma 2.3,

$$
|R|_{L^{\infty}(Q)} \leqslant C_{19}(\varepsilon) \delta
$$

if $\varepsilon \in\left(0, C_{19}^{-1}\right]$ and $\delta \in\left(0, C_{19}^{-1}(\varepsilon)\right]$ for some $C_{19}>0$ and some $C_{19}(\varepsilon)>$ 0 , which is stronger than (2.121). Let us remark also that, still if (2.119) holds, one does not need (2.11) of Proposition 2.1 and that Proposition 2.1 with $(2.11)$ omitted is already proved in [8]. Note that $(2.119)$ holds if $\Omega$ is the ball of radius $2(1-\bar{\sigma}) / \bar{\sigma}$ and that - take $\tilde{\sigma}=\sigma=0$ in the proof given in the next section - Theorem 1.1 still holds even with (2.118) required if, more generally,

$$
\sigma=0 \text { in } \Gamma \backslash \Gamma^{\#} .
$$

b) The reason for which we have not been able to treat the no-slip boundary condition (1.4) is that, with this boundary condition, we have not been able to get good enough estimates on $\omega$.

\section{Proof of Theorem 1.1 When $\Gamma^{\#} \neq \emptyset$}

Throughout all this section, we assume that

$$
\Gamma^{\#} \neq \emptyset \text {. }
$$

Let $y_{0} \in C^{\infty}\left(\bar{\Omega} ; \mathbb{R}^{2}\right)$ and $y_{1} \in C^{\infty}\left(\bar{\Omega} ; \mathbb{R}^{2}\right)$ be two maps such that (1.6), (1.7), (1.8), (1.9), and (1.10) hold; note that we do not assume that (1.11) holds. Let $\tilde{\Omega}$ be a bounded nonempty connected open subset of $\mathbb{R}^{2}$ of class $C^{\infty}$, let $\tilde{y}_{0} \in C^{\infty}\left(\overline{\tilde{\Omega}} ; \mathbb{R}^{2}\right)$ and let $\tilde{y}_{1} \in C^{\infty}\left(\overline{\tilde{\Omega}} ; \mathbb{R}^{2}\right)$ satisfying

$$
\begin{gathered}
\Omega \subset \tilde{\Omega}, \\
\left(\Gamma \backslash \Gamma^{\#}\right) \subset \tilde{\Gamma}:=\partial \tilde{\Omega}, \\
\Gamma^{\#} \subset \tilde{\Omega} \\
\operatorname{div} \tilde{y}_{0}=0 \text { in } \bar{\Omega} \\
\operatorname{div} \tilde{y}_{1}=0 \text { in } \overline{\tilde{\Omega}} \\
y_{0}=\tilde{y}_{0} \text { in } \bar{\Omega} \\
y_{1}=\tilde{y}_{1} \text { in } \bar{\Omega} .
\end{gathered}
$$

One easily checks that such $\tilde{\Omega}, \tilde{y}_{0}, \tilde{y}_{1}$ exist. Let $\tilde{\sigma} \in C^{\infty}(\tilde{\Gamma} ; \mathbb{R})$ be such that

$$
\tilde{\sigma}=\sigma \text { in } \Gamma \backslash \Gamma^{\#} \text {. }
$$


Fix some $T>0$ and take a function $\chi \in C^{\infty}(\mathbb{R} ; \mathbb{R})$ such that

$$
\begin{gathered}
\chi=1 \text { in }(-\infty, T / 4], \\
\chi=0 \text { in }[T / 2,+\infty) .
\end{gathered}
$$

Let $y^{\prime} \in C^{\infty}\left(\overline{\tilde{\Omega}} \times[0, T] ; \mathbb{R}^{2}\right)$ and $p^{\prime} \in C^{\infty}(\overline{\tilde{\Omega}} \times[0, T] ; \mathbb{R})$ be such that

$$
\frac{\partial y^{\prime}}{\partial t}-\Delta y^{\prime}+\left(y^{\prime} \cdot \nabla\right) y^{\prime}+\nabla p^{\prime}=0 \text { in } \overline{\tilde{\Omega}} \times[0, T] \text {, }
$$

$$
\operatorname{div} y^{\prime}=0 \text { in } \overline{\tilde{\Omega}} \times[0, T]
$$

$$
\begin{aligned}
y^{\prime}(x, t) \cdot \tilde{n}(x)=\chi(t) \tilde{y}_{0} \cdot \tilde{n}(x) & \\
& +(1-\chi(t)) \tilde{y}_{1} \cdot \tilde{n}(x), \forall(x, t) \in \tilde{\Gamma} \times[0, T],
\end{aligned}
$$

$$
\begin{gathered}
\tilde{\sigma}(x) y^{\prime}(x, t) \cdot \tilde{\tau}(x)+\operatorname{curl} y^{\prime}(x, t)=\chi(t)\left(\tilde{\sigma}(x) \tilde{y}_{0}(x) \cdot \tilde{\tau}(x)\right. \\
\left.+\operatorname{curl} \tilde{y}_{0}(x)\right), \forall(x, t) \in \tilde{\Gamma} \times[0, T], \\
y^{\prime}(\cdot, 0)=\tilde{y}_{0} \text { in } \overline{\tilde{\Omega}},
\end{gathered}
$$

where $\tilde{n}$ denotes the unit outward normal vector field to the boundary of $\tilde{\Omega}$ and where $\tilde{\tau}$ denotes the unit tangent vector field on $\tilde{\Gamma}$ such that $(\tilde{\tau}, \tilde{n})$ is a direct basis of $\mathbb{R}^{2}$. Let $K$ be a compact subset of $\Omega \cup \Gamma^{\#}$. By (3.2) and (3.4), $K$ is a (compact) subset of $\tilde{\Omega}$. Let us point out that in the previous section (1.11) was not used. We apply the result of this previous section with $\tilde{\Omega}$ instead of $\Omega, \tilde{\Omega} \backslash \bar{\Omega}$ instead of $\Omega^{\#}, y^{\prime}(T / 2)$ instead of $y_{0}, \tilde{y}_{1}$ instead of $y_{1},[T / 2, T]$ instead of $[0, T]$, and $\tilde{y}_{1} \cdot \tilde{n}$ instead of $\zeta$. Then it follows that, given $\nu>0$ and $\mu>0$, there exist $y^{\prime \prime} \in C^{\infty}\left(\overline{\tilde{\Omega}} \times[T / 2, T] ; \mathbb{R}^{2}\right)$ and $p^{\prime \prime} \in C^{\infty}(\overline{\tilde{\Omega}} \times[T / 2, T] ; \mathbb{R})$ such that

$$
\begin{aligned}
& \text { Support }\left(\frac{\partial y^{\prime \prime}}{\partial t}-\Delta y^{\prime \prime}+\left(y^{\prime \prime} \cdot \nabla\right) y^{\prime \prime}+\nabla p^{\prime \prime}\right) \subset(\tilde{\Omega} \backslash \bar{\Omega}) \times\left(\frac{T}{2}, T\right] \text {, } \\
& \operatorname{div} y^{\prime \prime}=0 \text { in } \bar{\Omega} \times\left[\frac{T}{2}, T\right], \\
& y^{\prime \prime}(x, t) \cdot \tilde{n}(x)=\tilde{y}_{1} \cdot \tilde{n}(x), \forall(x, t) \in \tilde{\Gamma} \times\left[\frac{T}{2}, T\right], \\
& \tilde{\sigma} y^{\prime \prime} \cdot \tilde{\tau}+\operatorname{curl} y^{\prime \prime}=0 \text { on } \tilde{\Gamma} \times\left[\frac{T}{2}, T\right] \text {, } \\
& y^{\prime \prime}\left(\cdot, \frac{T}{2}\right)=y^{\prime}\left(\cdot, \frac{T}{2}\right) \text { on } \overline{\tilde{\Omega}} \\
& \int_{\tilde{\Omega}} p^{\prime \prime}\left(\cdot, \frac{T}{2}\right)=\int_{\tilde{\Omega}} p^{\prime}\left(\cdot, \frac{T}{2}\right) \text {, } \\
& \left|y^{\prime \prime}(\cdot, T)-\tilde{y}_{1}\right|_{L^{\infty}(K)}+\left|\operatorname{curl} y^{\prime \prime}(\cdot, T)-\operatorname{curl} \tilde{y}_{1}\right|_{L^{\infty}(K)}<\nu, \\
& \left|y^{\prime \prime}(\cdot, T)-\tilde{y}_{1}\right|_{W^{-1, \infty}(\tilde{\Omega})}<\nu,
\end{aligned}
$$




$$
\left|\tilde{d}^{\mu}\left(y^{\prime \prime}(\cdot, T)-\tilde{y}_{1}\right)\right|_{L^{1}(\tilde{\Omega})}<\nu,
$$

where $\tilde{d}=\operatorname{dist}(\cdot, \tilde{\Gamma})$. Let $\tilde{y}: \overline{\widetilde{\Omega}} \times[0, T] \rightarrow \mathbb{R}^{2}$ be defined by

$$
\begin{aligned}
& \tilde{y}=y^{\prime} \text { in } \overline{\tilde{\Omega}} \times\left[0, \frac{T}{2}\right), \\
& \tilde{y}=y^{\prime \prime} \text { in } \overline{\widetilde{\Omega}} \times\left[\frac{T}{2}, T\right],
\end{aligned}
$$

and let $\tilde{p}: \bar{\Omega} \times[0, T] \rightarrow \mathbb{R}$ be defined by

$$
\begin{aligned}
& \tilde{p}=p^{\prime} \text { in } \overline{\tilde{\Omega}} \times\left[0, \frac{T}{2}\right), \\
& \tilde{p}=p^{\prime \prime} \text { in } \bar{\Omega} \times\left[\frac{T}{2}, T\right] .
\end{aligned}
$$

From (3.10) to (3.14), (3.16) to (3.21), and (3.25) to (3.28), we get that $\tilde{y}$ and $\tilde{p}$ are of class $C^{\infty}$. Let us define $y \in C^{\infty}\left(\bar{\Omega} \times[0, T] ; \mathbb{R}^{2}\right)$ and $p \in$ $C^{\infty}(\bar{\Omega} \times[0, T] ; \mathbb{R})$ by

$$
\begin{aligned}
& y=\tilde{y} \text { in } \bar{\Omega} \times[0, T], \\
& p=\tilde{p} \text { in } \bar{\Omega} \times[0, T] .
\end{aligned}
$$

From (3.2), (3.11), (3.16), (3.25), (3.26), (3.27), (3.28), (3.29), and (3.30), we get

$$
\frac{\partial y}{\partial t}-\Delta y+(y \cdot \nabla) y+\nabla p=0 \text { in } \bar{\Omega} \times[0, T]
$$

from (3.2), (3.12), (3.17), (3.25), (3.26), and (3.29), we get (1.13); from (1.8), (1.9), (3.3), (3.14), (3.18), (3.25), (3.26), and (3.29), we get (1.14); from (1.10), (3.9), (3.14), (3.19), (3.25), (3.26), and (3.29), we get (1.15). From (3.2), (3.8), (3.15), (3.25), and (3.29), we get (1.16). From (3.2), (3.8), $(3.22),(3.23),(3.26)$, and $(3.29)$, we get

$$
\begin{gathered}
\left|y(\cdot, T)-y_{1}\right|_{L^{\infty}(K)}+\left|\operatorname{curl} y(\cdot, T)-\operatorname{curl} y_{1}\right|_{L^{\infty}(K)}<\nu, \\
\left|y(\cdot, T)-y_{1}\right|_{W^{-1, \infty}(\Omega)}<\nu .
\end{gathered}
$$

Note that, by (3.2),

$$
d \leqslant \tilde{d} \text { in } \Omega,
$$

which, with (3.2), (3.8), (3.24), (3.26), and (3.29), implies that

$$
\left|d^{\mu}\left(y(\cdot, T)-y_{1}\right)\right|_{L^{1}(\Omega)}<\nu .
$$

This ends the proof of Theorem 1.1.

Remark 3.1. Theorem 1.1 still holds if one assumes $y_{0}$ to be of class $C^{1}$ provided that, instead of requiring that $\left(y^{k}, p^{k}\right)$ are of class $C^{\infty}$ on $[0, T] \times \bar{\Omega}$, one requires only that they are of class $C^{\infty}$ on $(0, T] \times \bar{\Omega}$ and continuous on $[0, T] \times \bar{\Omega}$ and in (1.12), (1.13), and (1.15) one replaces $[0, T]$ by $(0, T]$. This follows directly from our proof when $\Gamma^{\#}=\emptyset$. When $\Gamma^{\#} \neq \emptyset$, it suffices to now choose $\tilde{y}_{0}$ to be of class $C^{1}$ only and to replace (3.13) and (3.14) by

$$
\begin{gathered}
y^{\prime}(x, t) \cdot \tilde{n}(x)=\chi_{0}(x, t), \forall(x, t) \in \tilde{\Gamma} \times[0, T], \\
\tilde{\sigma}(x) y^{\prime}(x, t) \cdot \tilde{\tau}(x)+\operatorname{curl} y^{\prime}(x, t)=\chi_{1}(x, t), \forall(x, t) \in \tilde{\Gamma} \times[0, T], \\
\text { Esaim: Cocv, MaY 1996, Vol. } 1, \text { PF. } 35-75 .
\end{gathered}
$$


where $\chi_{0}$ and $\chi_{1}$ are such that

$$
\begin{gathered}
\chi_{0} \in C^{1}(\overline{\tilde{\Omega}} \times[0, T] ; \mathbb{R}), \\
\chi_{0} \in C^{\infty}(\overline{\tilde{\Omega}} \times(0, T] ; \mathbb{R}), \\
\chi_{1} \in C^{0}(\overline{\tilde{\Omega}} \times[0, T] ; \mathbb{R}), \\
\chi_{1} \in C^{\infty}(\overline{\tilde{\Omega}} \times(0, T] ; \mathbb{R}), \\
\chi_{0}(x, 0)=\tilde{y_{0}}(x) \cdot \tilde{n}(x), \forall x \in \tilde{\Gamma}, \\
\left.\chi_{1}(x, 0)=\tilde{\sigma}(x) \tilde{y_{0}}(x) \cdot \tilde{\tau}(x)+\operatorname{curl} \tilde{y_{0}}(x), \forall x, t\right) \in \tilde{\Gamma}, \\
\chi_{0}(x, t)=\tilde{y_{1}}(x) \cdot \tilde{n}(x), \forall(x, t) \in \tilde{\Gamma} \times[T / 2, T], \\
\chi_{1}(x, t)=\tilde{\sigma}(x) \tilde{y_{1}}(x) \cdot \tilde{\tau}(x)+\operatorname{curl} \tilde{y_{1}}(x), \forall(x, t) \in \tilde{\Gamma} \times[T / 2, T] .
\end{gathered}
$$

In fact one can consider $y_{0}$ which are even less regular. Indeed, using the class of weak solutions of the Navier-Stokes equations introduced by J.-L. Lions in [18, Chapitre 1 , Théorème 6.10$]$, one sees that it is enough to assume that $y_{0} \in H^{1}(\Omega)$ and curl $y_{0} \in L^{\infty}(\Omega)$.

Acknowledgments. This work was completed while the author was visiting the University of Bonn. We are very thankful to S. Hildebrandt for his hospitality and interesting discussions. We also thank H.W. Alt, C. Bardos, E. Bäusch, E. Fernández-Cara, G. Geymonat, J.-M. Ghidaglia, O. Pironneau, J. Real, and E. Zuazua for useful discussions, comments or references.

\section{Appendix A. Proof of Proposition 2.1}

Let us first recall that, with condition (2.11) omitted, this proposition is already proved in $[8]$. For $X \in C^{\infty}\left(\bar{\Omega} \times\left[T_{1}, T_{2}\right] ; \mathbb{R}^{2}\right)$ such that

$$
X \cdot n=0 \text { on } \Gamma \times\left[T_{1}, T_{2}\right],
$$

let us denote by $\phi^{X}$ the flow associated to $X$, i.e. the map $\phi^{X}$ in $C^{\infty}(\bar{\Omega} \times$ $\left.\left[T_{1}, T_{2}\right] \times\left[T_{1}, T_{2}\right] ; \bar{\Omega}\right),\left(x, t_{1}, t_{2}\right) \rightarrow \phi^{X}\left(x, t_{1}, t_{2}\right)$ defined by

$$
\begin{gathered}
\frac{\partial \phi^{X}}{\partial t_{1}}=X\left(\phi^{X}, t_{1}\right), \\
\phi^{X}(x, t, t)=x, \forall x \in \bar{\Omega}, \forall t \in\left[t_{1}, t_{2}\right] .
\end{gathered}
$$

Let us denote by $B(x, r)$ the closed ball in $\mathbb{R}^{2}$ of radius $r$ centered at $x \in \mathbb{R}^{2}$. Reducing $\Omega^{\#}$, if necessary, we may assume that $\Omega^{\#}$ is a nonempty open subset of $\mathbb{R}^{2}$ of class $C^{\infty}$ satisfying (2.5) and such that

$$
\Omega^{\#} \text { is connected and simply connected. }
$$

We will use the following lemma, whose proof is deferred.

Lemma A.1. For any $x$ in $\Omega$, there exists $r(x) \in(0, d(x))$ such that, for any $\varepsilon>0$, there exists $\theta^{\varepsilon, x}$ in $C^{\infty}(\bar{\Omega} \times[0,1] ; \mathbb{R})$ such that

$$
\begin{gathered}
\theta^{\varepsilon, x}=0 \text { in } \bar{\Omega} \times\left(\left[0, \frac{1}{4}\right] \cup\left[\frac{3}{4}, 1\right]\right), \\
\Delta \theta^{\varepsilon, x}=0 \text { in }\left(\bar{\Omega} \backslash \Omega^{\#}\right) \times[0,1], \\
\frac{\partial \theta^{\varepsilon, x}}{\partial n}=0 \text { on } \Gamma \times[0,1],
\end{gathered}
$$




$$
\begin{gathered}
\left|\frac{\partial \theta^{\varepsilon, x}}{\partial \tau}\right|+\left|\frac{\partial^{2} \theta^{\varepsilon, x}}{\partial \tau^{2}}\right| \leqslant \varepsilon \text { on } \Gamma \times[0,1], \\
\phi^{\nabla \theta^{\varepsilon, x}}(B(x, r(x)), 1,0) \subset \Omega^{\#} .
\end{gathered}
$$

Let us fix $\bar{\eta}>0$ so that the following property holds:

$(\mathcal{P})$ for all $x$ in $\bar{\Omega}$ with $d(x)<\bar{\eta}$, there exists a unique $P(x) \in \Gamma$ such that $d(x)=|x-P(x)|$ and, on $\{x \in \bar{\Omega} ; d(x)<\bar{\eta}\}$, application $P$ is of class $C^{\infty}$.

Let us recall that we denote by $\Gamma_{0}, \ldots, \Gamma_{g}$ the connected components of $\Gamma$. Note that $(\mathcal{P})$ implies that, for all $i \in[0, g]$ and all $j \in[0, g]$ with $i \neq j$,

$$
\left\{x \in \bar{\Omega} ; \operatorname{dist}\left(x, \Gamma_{i}\right)<\bar{\eta}\right\} \cap\left\{x \in \Omega ; \operatorname{dist}\left(x, \Gamma_{j}\right)<\bar{\eta}\right\}=\emptyset \text {. }
$$

Let

$$
\varepsilon \in(0, \bar{\eta})
$$

and let

$$
K_{0}=\{x \in \bar{\Omega} ; d(x) \geqslant \varepsilon\} .
$$

We assume $\varepsilon>0$ small enough so that - recall $(2.5)-$

$$
\Omega^{\#} \subset K_{0} \text {. }
$$

Let $x_{1}, \ldots, x_{j}, \ldots, x_{m}$ be a finite sequence of points in $\Omega$ such that

$$
K_{0} \subset \cup_{j=1}^{m} B\left(x_{j}, r\left(x_{j}\right)\right) .
$$

Let $t_{0}=T / 2$, let $t_{m+(1 / 2)}=T$. For $j \in[1, m]$, let

$$
\begin{gathered}
t_{j-\frac{1}{2}}=t_{0}+(j-1) \frac{T}{2 m}+\frac{T}{4 m}, \\
t_{j}=t_{j-\frac{1}{2}}+\frac{T}{4 m}=t_{0}+j \frac{T}{2 m} .
\end{gathered}
$$

Let $\varepsilon>0$. Let $\theta^{\varepsilon} \in C^{\infty}(\bar{\Omega} \times[0, T] ; \mathbb{R})$ be defined by

$$
\theta^{\varepsilon}=0 \text { in } \bar{\Omega} \times\left[0, t_{0}\right]
$$

and by, for all $j \in[1, m]$,

$$
\begin{gathered}
\theta^{\varepsilon}(x, t)=\frac{4 m}{T} \theta^{\frac{\varepsilon T}{4 m}, x_{j}}\left(x, \frac{4 m}{T}\left(t-t_{j-1}\right)\right), \forall(x, t) \in \bar{\Omega} \times\left[t_{j-1}, t_{j-\frac{1}{2}}\right], \\
\theta^{\varepsilon}(x, t)=-\frac{4 m}{T} \theta^{\frac{\varepsilon T}{4 m}, x_{j}}\left(x, \frac{4 m}{T}\left(t_{j}-t\right)\right), \forall(x, t) \in \bar{\Omega} \times\left[t_{j-\frac{1}{2}}, t_{j}\right] .
\end{gathered}
$$

Then - see in particular (A.2) to (A.5) - (2.7), (2.8), (2.9), and (2.11) are satisfied. Note that, by $(2.7),(2.8),(2.9)$, and (A.1), there exists $\psi^{\varepsilon}$ in $C^{\infty}(\bar{\Omega} \times[0, T] ; \mathbb{R})$ such that

$$
\nabla \psi^{\varepsilon}=-\nabla^{\perp} \theta^{\varepsilon} \text { in }\left(\bar{\Omega} \backslash \Omega^{\#}\right) \times[0, T]
$$

$$
\text { Support } \psi^{\varepsilon} \subset \bar{\Omega} \times[T / 4, T) \text {. }
$$

Let

$$
\bar{y}^{\varepsilon}=\nabla^{\perp} \psi^{\varepsilon} .
$$

Then (2.10), (2.12), and (2.13) hold. 
The only step that remains is to construct $Z^{\varepsilon}$ and $\Pi^{\varepsilon}$. Let $z_{0} \in C^{\infty}\left(\bar{\Omega} ; \mathbb{R}^{2}\right)$ and $z_{1} \in C^{\infty}\left(\bar{\Omega} ; \mathbb{R}^{2}\right)$ be such that (2.14) to (2.17) hold. For simplicity omit the index $\varepsilon$. Let $\omega^{0} \in C^{\infty}(\bar{\Omega} \times[0, T] ; \mathbb{R})$ be defined by

$$
\begin{gathered}
\frac{\partial \omega^{0}}{\partial t}+(\nabla \theta \cdot \nabla) \omega^{0}=0 \text { in } \bar{\Omega} \times[0, T], \\
\omega^{0}(\cdot, 0)=\operatorname{curl} z_{0} \text { in } \bar{\Omega} .
\end{gathered}
$$

Let $\psi^{*} \in C^{\infty}(\bar{\Omega} ; \mathbb{R})$ be defined by

$$
\begin{gathered}
\Delta \psi^{*}=\omega^{0}(\cdot, T) \text { in } \bar{\Omega}, \\
\psi^{*}=0 \text { on } \Gamma .
\end{gathered}
$$

Let $\bar{b} \in C^{\infty}(\mathbb{R} ;[0,1])$ be such that

$$
\begin{aligned}
& \bar{b}=1 \text { in }\left(-\infty, \frac{1}{2}\right], \\
& \bar{b}=0 \text { in }\left[\frac{3}{4},+\infty\right) ;
\end{aligned}
$$

let $b \in C^{\infty}(\bar{\Omega} ; \mathbb{R})$ and, for $i \in[0, g]$, let $b_{i} \in C^{\infty}(\bar{\Omega} ; \mathbb{R})$ be defined by

$$
\begin{gathered}
b(x)=\bar{b}\left(\frac{d(x)}{\varepsilon}\right), \forall x \in \bar{\Omega}, \\
b^{i}(x)=\bar{b}\left(\frac{\operatorname{dist}\left(x, \Gamma_{i}\right)}{\varepsilon}\right), \forall x \in \bar{\Omega} .
\end{gathered}
$$

Let $\xi^{0} \in C^{\infty}(\bar{\Omega} ; \mathbb{R})$ be such that

$$
\begin{gathered}
\text { Support } \Delta \xi^{0} \subset \Omega^{\#}, \\
\int_{\Omega} \Delta \xi^{0} \neq 0, \\
\xi^{0}=1 \text { on } \Gamma_{0}, \\
\xi^{0}=0 \text { on } \Gamma \backslash \Gamma_{0} .
\end{gathered}
$$

Let $A=\left(A_{i j} ; 0 \leqslant i \leqslant g, 0 \leqslant j \leqslant g\right)$ be the matrix defined by

$$
A_{i j}=\int_{\Gamma_{i}} \frac{\partial \xi^{j}}{\partial n}, \forall(i, j) \in[0, g]^{2} .
$$

Let us check that

$$
A \text { is invertible. }
$$

First, let us point out that, by (2.99), (2.100), (2.101), and (A.28),

$$
A_{i j}=\int_{\Omega} \nabla \xi^{i} \nabla \xi^{j}, \forall(i, j) \in[1, g]^{2} .
$$

Hence, by (2.109), if (A.29) does not hold, there exists $\left(a_{1}, \ldots, a_{g}\right) \in \mathbb{R}^{g}$ such that

$$
\int_{\Gamma_{i}} \frac{\partial \xi^{0}}{\partial n}=\sum_{j=1}^{g} a_{j} \int_{\Gamma_{i}} \frac{\partial \xi^{j}}{\partial n}, \forall i \in[0, g],
$$


which gives

$$
\int_{\Omega} \Delta \xi^{0}=\int_{\Gamma} \frac{\partial \xi^{0}}{\partial n}=\sum_{j=1}^{g} a_{j} \int_{\Gamma} \frac{\partial \xi^{j}}{\partial n}=\sum_{j=1}^{g} a_{j} \int_{\Omega} \Delta \xi^{j} .
$$

But (A.31) is in contradiction with (2.99) and (A.25). Hence (A.29) holds. Let $\psi^{0} \in C^{\infty}(\bar{\Omega} ; \mathbb{R})$ be such that

$$
\begin{gathered}
\Delta \psi^{0}=\operatorname{curl} z_{0} \text { in } \bar{\Omega}, \\
\psi^{0}=0 \text { on } \Gamma,
\end{gathered}
$$

let $\Xi \in C^{\infty}(\bar{\Omega} ; \mathbb{R})$ be such that

$$
\begin{aligned}
& \Delta \Xi=0 \text { in } \bar{\Omega}, \\
& \frac{\partial \Xi}{\partial n}=\zeta \text { on } \Gamma ;
\end{aligned}
$$

by (2.1) such a $\Xi$ exists. Then there exists a (unique) $\left(\lambda_{1}^{0}, \ldots, \lambda_{g}^{0}\right)$ such that

$$
z_{0}=\nabla^{\perp} \psi^{0}+\nabla \Xi+\sum_{j=1}^{g} \lambda_{i}^{0} \nabla^{\perp} \xi^{i} .
$$

Similarly, let $\psi^{1} \in C^{\infty}(\bar{\Omega} ; \mathbb{R})$ be such that

$$
\begin{gathered}
\Delta \psi^{1}=\operatorname{curl} z_{1} \text { in } \bar{\Omega}, \\
\psi^{1}=0 \text { on } \Gamma,
\end{gathered}
$$

and let $\left(\lambda_{1}^{1}, \ldots, \lambda_{g}^{1}\right)$ be such that

$$
z_{1}=\nabla^{\perp} \psi^{1}+\nabla \Xi+\sum_{j=1}^{g} \lambda_{i}^{1} \nabla^{\perp} \xi^{i}
$$

By (A.29), there exists one (and only one) $\left(\mu_{0}, \ldots, \mu_{g}\right) \in \mathbb{R}^{g+1}$ such that

$$
\sum_{j=0}^{g} A_{i j} \mu_{j}=-\sum_{j=1}^{g} A_{i j}\left(\lambda_{j}^{0}-\lambda_{j}^{1}\right)+\int_{\Gamma_{i}} \frac{\partial \psi^{*}}{\partial n}-\int_{\Gamma_{i}} \frac{\partial \psi^{0}}{\partial n}, \forall i \in[0, g] .
$$

We define $\tilde{\psi} \in C^{\infty}(\bar{\Omega} ; \mathbb{R})$ by

$$
\tilde{\psi}=b \psi^{*}+(1-b) \psi_{1}+\sum_{i=0}^{g} \mu_{i}\left(1-\xi^{i}\right) b^{i}
$$

and $\tilde{\omega} \in C^{\infty}(\bar{\Omega} \times[0, T] ; \mathbb{R})$ by

$$
\begin{gathered}
\frac{\partial \tilde{\omega}}{\partial t}+(\nabla \theta \cdot \nabla) \tilde{\omega}=0 \text { in } \bar{\Omega} \times[0, T], \\
\tilde{\omega}(\cdot, T)=\Delta \tilde{\psi} \text { in } \bar{\Omega} .
\end{gathered}
$$

By (A.6), there exists $\tilde{\eta}>0$ such that, for all $j \in[1, m]$ and for all $x$ in $B\left(x_{j}, r\left(x_{j}\right)\right)$,

$$
\operatorname{dist}\left(\phi^{\nabla \theta^{\frac{\varepsilon T}{4 m}, x_{j}}}(x, 1,0), \bar{\Omega} \backslash \Omega^{\#}\right) \geqslant \tilde{\eta}
$$


Let $b^{\#} \in C^{\infty}(\bar{\Omega} ; \mathbb{R})$ be such that

$$
\begin{gathered}
\text { Support } b^{\#} \subset \Omega^{\#}, \\
b^{\#}(x)=1, \forall x \in \Omega^{\#} \text { such that } \operatorname{dist}\left(x, \bar{\Omega} \backslash \Omega^{\#}\right) \geqslant \tilde{\eta} .
\end{gathered}
$$

Let $g \in C^{\infty}(\mathbb{R} ; \mathbb{R})$ be such that

$$
\begin{aligned}
& g=0 \text { in }\left(-\infty, \frac{T}{21 m}\right], \\
& g=1 \text { in }\left[\frac{T}{20 m},+\infty\right) .
\end{aligned}
$$

For $j \in[1, m]$, we define by induction on $j, \omega^{j} \in C^{0}\left(\bar{\Omega} \times\left[t_{j-(1 / 2)}, t_{j+(1 / 2)}\right] ; \mathbb{R}\right)$ by requiring

$$
\begin{aligned}
\omega^{j}(x, t)=\left(1-g\left(t-t_{j-\frac{1}{2}}\right) b^{\#}(x)\right) \omega^{j-1}\left(x, t_{j-\frac{1}{2}}\right) & \\
& +g\left(t-t_{j-\frac{1}{2}}\right) b^{\#}(x) \tilde{\omega}(x, t),
\end{aligned}
$$

for all $(x, t)$ in $\bar{\Omega} \times\left[t_{j-(1 / 2)}, t_{j-(1 / 2)}+(T /(20 m))\right]$ and

$$
\frac{\partial \omega^{j}}{\partial t}+(\nabla \theta \cdot \nabla) \omega^{j}=0 \text { in } \Omega \times\left(t_{j-\frac{1}{2}}+\frac{T}{20 m}, t_{j+\frac{1}{2}}\right) .
$$

Using (A.2), (A.13), (A.15), (A.42), (A.48), (A.49), and (A.50), one sees that $\omega^{j}$ is of class $C^{\infty}$ on $\bar{\Omega} \times\left[t_{j-(1 / 2)}, t_{j+(1 / 2)}\right]$. Let $\omega: \bar{\Omega} \times[0, T] \rightarrow \mathbb{R}$ be defined by

$$
\begin{gathered}
\omega=\omega^{0} \text { in } \bar{\Omega} \times\left[0, t_{0}\right], \\
\omega=\omega^{j} \text { in } \bar{\Omega} \times\left(t_{j-\frac{1}{2}}, t_{j+\frac{1}{2}}\right], \forall j \in[1, m] .
\end{gathered}
$$

Again one easily checks that $\omega$ is of class $C^{\infty}$. Moreover, by (A.17) and (A.51), it satisfies

$$
\omega(\cdot, 0)=\operatorname{curl} z_{0} .
$$

By (2.12), (A.2), (A.12), (A.13), (A.15), (A.16), (A.45), (A.49), (A.50), (A.51), and (A.52), one has

$$
\frac{\partial \omega}{\partial t}+(\bar{y} \cdot \nabla) \omega=0 \text { in }\left(\bar{\Omega} \backslash \Omega^{\#}\right) \times[0, T] .
$$

We claim that

$$
\omega(\bar{x}, T)=\tilde{\omega}(\bar{x}, T), \forall \bar{x} \in \Omega .
$$

Let us first consider the case where $d(\bar{x}) \geqslant \frac{\varepsilon}{2}$. Then, by (A.9), (A.11), (A.44), and (A.46), there exists $j \in[1, m]$ such that

$$
b^{\#}\left(\phi^{\nabla \theta^{\frac{\varepsilon T}{4 m}, x_{j}}}(\bar{x}, 1,0)\right)=1 .
$$

From (A.13), (A.50), and (A.52), we get

$$
\omega\left(\bar{x}, t_{j}\right)=\omega^{j}\left(\phi^{\nabla \theta}\left(\bar{x}, t_{j-\frac{1}{2}}+\frac{T}{20 m}, t_{j}\right), t_{j-\frac{1}{2}}+\frac{T}{20 m}\right) .
$$


But, using (A.2), (A.13), and (A.15), we have

$$
\phi^{\nabla \theta}\left(\bar{x}, t_{j-\frac{1}{2}}+\frac{T}{20 m}, t_{j}\right)=\phi^{\nabla \theta^{\frac{\varepsilon T}{4 m}, x_{j}}}(\bar{x}, 1,0),
$$

which, with (A.12), (A.48), (A.49), (A.56), and (A.57) implies that

$$
\omega\left(\bar{x}, t_{j}\right)=\tilde{\omega}\left(\phi^{\nabla \theta}\left(\bar{x}, t_{j-\frac{1}{2}}+\frac{T}{20 m}, t_{j}\right), t_{j-\frac{1}{2}}\right) .
$$

But, by (A.2), (A.13), (A.14), and (A.42),

$$
\begin{aligned}
\tilde{\omega}\left(\phi^{\nabla \theta}\left(\bar{x}, t_{j-\frac{1}{2}}+\frac{T}{20 m}, t_{j}\right), t_{j-\frac{1}{2}}\right) & =\tilde{\omega}\left(\phi^{\nabla \theta}\left(\bar{x}, t_{j-\frac{1}{2}}, t_{j}\right), t_{j-\frac{1}{2}}\right) \\
& =\tilde{\omega}\left(\bar{x}, t_{j}\right),
\end{aligned}
$$

which, with (A.58), gives

$$
\omega\left(\bar{x}, t_{j}\right)=\tilde{\omega}\left(\bar{x}, t_{j}\right) .
$$

Moreover, from (A.12), (A.13), (A.14), and (A.15), we get

$$
\phi^{\nabla \theta}\left(x, t_{i-1}, t_{i}\right)=x, \forall x \in \bar{\Omega}, \forall i \in[1, m],
$$

which, with (A.2), (A.12), (A.13), (A.15), (A.42), (A.49), (A.50), and (A.52), implies that for all $i \in[1, m]$ and all $x$ in $\bar{\Omega}$,

$$
\omega\left(x, t_{i-1}\right)=\tilde{\omega}\left(x, t_{i-1}\right) \Rightarrow \omega\left(x, t_{i}\right)=\tilde{\omega}\left(x, t_{i}\right) .
$$

From (A.13), (A.59), and (A.60), we get

$$
\omega(\bar{x}, T)=\tilde{\omega}(\bar{x}, T) .
$$

Let us now study the case where $d(\bar{x})<\varepsilon / 2$. Then, by (2.99), (A.7), (A.8), (A.9), (A.10), (A.18), (A.20), (A.21), (A.22), (A.23), (A.24), (A.41), and (A.43),

$$
\tilde{\omega}(\bar{x}, T)=\omega^{0}(\bar{x}, T),
$$

which, with (A.16) and (A.42), implies that

$$
\tilde{\omega}\left(\phi^{\nabla \theta}(\bar{x}, t, T), t\right)=\omega^{0}\left(\phi^{\nabla \theta}(\bar{x}, t, T), t\right), \forall t \in[0, T] .
$$

In particular, by $(\mathrm{A} .51)$,

$$
\begin{aligned}
\tilde{\omega}\left(\phi^{\nabla \theta}\left(\bar{x}, t_{\frac{1}{2}}, T\right), t_{\frac{1}{2}}\right) & =\omega^{0}\left(\phi^{\nabla \theta}\left(\bar{x}, t_{\frac{1}{2}}, T\right), t_{\frac{1}{2}}\right) \\
& =\omega\left(\phi^{\nabla \theta}\left(\bar{x}, t_{\frac{1}{2}}, T\right), t_{\frac{1}{2}}\right),
\end{aligned}
$$

which, using again (A.2), (A.12), (A.13), (A.15), (A.42), (A.49), (A.50) and (A.52), implies that

$$
\omega(\bar{x}, T)=\tilde{\omega}(\bar{x}, T)
$$

Hence (A.55) holds.

Let $\psi \in C^{\infty}(\bar{\Omega} \times[0, T] ; \mathbb{R})$ be defined by

$$
\begin{gathered}
\Delta \psi=\omega \text { in } \bar{\Omega} \times[0, T], \\
\psi=0 \text { on } \Gamma \times[0, T] .
\end{gathered}
$$

From (A.32), (A.33), (A.53), (A.62), and (A.63), we get

$$
\psi(\cdot, 0)=\psi^{0} \text { in } \bar{\Omega} \text {. }
$$


Note also that, by (2.101), (A.7), (A.8), (A.19), (A.21), (A.23), (A.26), and (A.41),

$$
\tilde{\psi}=0 \text { on } \Gamma
$$

which, with (A.43), (A.55), (A.62), and (A.63), implies that

$$
\psi(\cdot, T)=\tilde{\psi} \text { in } \bar{\Omega} .
$$

Let $\left(\lambda_{i} ; i \in[0, g]\right) \in C^{\infty}([0, T] ; \mathbb{R})^{g+1}$ be such that (see $($ A.29))

$$
\begin{gathered}
\sum_{j=0}^{g} A_{i j} \frac{d \lambda_{j}}{d t}=\frac{d}{d t} \int_{\Gamma_{i}} \nabla^{\perp} \psi \cdot \tau, \forall i \in[0, g], \\
\lambda_{j}(0)=\lambda_{j}^{0}, \forall j \in[1, g], \\
\lambda_{0}(0)=0 .
\end{gathered}
$$

Finally, let $z \in C^{\infty}\left(\bar{\Omega} \times[0, T] ; \mathbb{R}^{2}\right)$ be defined by

$$
z=\nabla^{\perp} \psi+\nabla \Xi+\sum_{j=0}^{g} \lambda_{j} \nabla^{\perp} \xi^{j} .
$$

From (A.29), (A.36), (A.51), (A.62), (A.63), (A.66), (A.67), and (A.69), one gets (2.20). From (A.34) and (A.69), one gets (2.22). From (2.100), (2.101), (A.26), (A.27), (A.35) (A.63), and (A.69), one gets (2.23). Straightforward estimations show that $(2.24)$ hold for $C^{*}$ large enough (independent of $z_{0}, z_{1}$ and $\varepsilon$.) Usual estimates give $(2.25)$ for $\bar{C}(\varepsilon)$ large enough but independent of $z_{0}$ and $z_{1}$. Let $\lambda_{0}^{0}=\lambda_{0}^{1}=0$. From (A.64), (A.65), (A.66) (A.67), and (A.68), one gets

$$
\sum_{j=0}^{g} A_{i j}\left(\lambda_{j}(T)-\lambda_{j}^{0}\right)=\int_{\Gamma_{i}} \frac{\partial \psi^{0}}{\partial n}-\int_{\Gamma_{i}} \frac{\partial \tilde{\psi}}{\partial n}, \forall i \in[0, g] .
$$

However, by (A.7), (A.8), (A.20), (A.21), (A.22), (A.23), (A.28), and (A.41),

$$
\begin{aligned}
\int_{\Gamma_{i}} \frac{\partial \tilde{\psi}}{\partial n} & =\int_{\Gamma_{i}} \frac{\partial \psi^{*}}{\partial n}-\sum_{i=0}^{g} \mu_{j} \int_{\Gamma_{i}} \frac{\partial \xi^{j}}{\partial n} \\
& =\int_{\Gamma_{i}} \frac{\partial \psi^{*}}{\partial n}-\sum_{i=0}^{g} A_{i j} \mu_{j},
\end{aligned}
$$

which, with (A.29), (A.40) and (A.70), implies that

$$
\lambda_{j}(T)=\lambda_{j}^{1}, \forall j \in[0, g] .
$$

From (A.21), (A.22), (A.23), and (A.41), we get

$$
\nabla \tilde{\psi}(x)=\nabla \psi^{1}(x), \forall x \in \bar{\Omega} \text { such that } d(x) \geqslant \varepsilon,
$$

which, with (A.39), (A.65), (A.69), and (A.71), implies (2.21).

It remains to check the existence of $\pi$ such that (2.18) and (2.19) hold. It follows from (2.12), (2.13), (2.22), (2.99), (A.24), (A.34), (A.54), (A.62), and (A.69) that

$$
\operatorname{curl}\left(\frac{\partial}{\partial t} z+\bar{y} \cdot \nabla z+z \cdot \nabla \bar{y}\right)=0 \text { in }\left(\bar{\Omega} \backslash \Omega^{\#}\right) \times[0, T] .
$$


Using (2.5), (2.7), (2.8), and (2.12), one gets

$$
\int_{\Gamma_{i}}(\bar{y} \cdot \nabla z+z \cdot \nabla \bar{y}) \cdot \tau=0, \forall i \in[0, g]
$$

and that, by (A.28), (A.66), and (A.69),

$$
\int_{\Gamma_{i}} \frac{\partial z}{\partial t} \cdot \tau=0, \forall i \in[0, g] \text {. }
$$

The existence of $\pi$ such that (2.18) and (2.19) hold follows from (2.10), (2.20), (A.1), (A.72), (A.73), and (A.74).

Let us now turn to the proof Lemma A.1. Let us first prove the following Lemma A.2. For any $\bar{x}$ in $\Omega$

$\left\{\nabla \theta(\bar{x}) ; \theta \in C^{\infty}(\bar{\Omega} ; \mathbb{R})\right.$ such that $\Delta \theta=0$ in $\bar{\Omega} \backslash \Omega^{\#}$ and $\frac{\partial \theta}{\partial n}=0$ on $\left.\Gamma\right\}=\mathbb{R}^{2}$.

Indeed, if this lemma does not hold, there exist $\bar{x}$ in $\Omega$ and $V$ in $\mathbb{R}^{2}$ such that

$$
V \neq 0
$$

and, for all $\theta$ in $C^{\infty}(\bar{\Omega} ; \mathbb{R})$ such that

$$
\Delta \theta=0 \text { in } \bar{\Omega} \backslash \Omega^{\#}
$$

and such that

one has

$$
\frac{\partial \theta}{\partial n}=0 \text { on } \Gamma
$$

$$
V \cdot \nabla \theta(\bar{x})=0 .
$$

Let $\bar{a} \in \Omega^{\#} \backslash\{\bar{x}\}$. For $a \in \Omega^{\#}$, let $\varphi^{a} \in L^{1}(\bar{\Omega}) \cap C^{\infty}(\bar{\Omega} \backslash\{a, \bar{a}\} ; \mathbb{R})$ be the solution of

$$
\begin{gathered}
\Delta \varphi^{a}=\delta_{a}-\delta_{\bar{a}} \text { in } \Omega, \\
\int_{\Omega} \varphi^{a}=0, \\
\frac{\partial \varphi^{a}}{\partial n}=0 \text { on } \Gamma .
\end{gathered}
$$

Then, an easy density argument shows that

$$
V \cdot \nabla \varphi^{a}(\bar{x})=0, \forall a \in \Omega^{\#} \backslash\{\bar{a}, \bar{x}\}
$$

and, so by analyticity of $a \in \Omega \backslash\{\bar{a}, \bar{x}\} \rightarrow \nabla \varphi^{a}(\bar{x})$ and by the connexity of $\Omega \backslash\{\bar{a}, \bar{x}\}$,

$$
V \cdot \nabla \varphi^{a}(\bar{x})=0, \forall a \in \Omega \backslash\{\bar{a}, \bar{x}\} .
$$

But usual estimates on the Green functions show that

$$
\nabla \varphi^{a}(\bar{x})=\frac{1}{2 \pi} \frac{\bar{x}-a}{|\bar{x}-a|^{2}}+O(1) \text { as } a \rightarrow \bar{x},
$$

which is in contradiction with (A.75) and (A.76). This finishes the proof of Lemma A.2.

Let now fix $x \in \Omega$. Let $F \in C^{\infty}([0,1] ; \Omega)$ be such that

$$
\begin{gathered}
F(0)=x, \\
F(1) \in \Omega^{\#},
\end{gathered}
$$


$F$ is an embedding.

Let $h \in C^{\infty}([0,1] ;[0,1])$ be such that

$$
\begin{aligned}
& h=0 \text { in }\left[0, \frac{1}{4}\right], \\
& h=1 \text { in }\left[\frac{3}{4}, 1\right],
\end{aligned}
$$

and let $\bar{F} \in C^{\infty}([0,1] ; \Omega)$ be defined by

$$
\bar{F}(t)=F \circ h(t), \forall t \in[0,1] .
$$

It follows easily from Lemma A.2, (A.77), (A.78), (A.80), (A.81), and (A.82) that there exist an integer $l, l$ many functions $h_{1}, \ldots, h_{l}$ in $C^{\infty}([0,1] ; \mathbb{R})$ and $l$ many functions $\bar{\theta}^{1}, \ldots, \bar{\theta}^{l}$ in $C^{\infty}(\bar{\Omega} ; \mathbb{R})$ such that

$$
\begin{gathered}
\text { Support } h_{i} \subset\left[\frac{1}{4}, \frac{3}{4}\right], \forall i \in[1, l], \\
\Delta \bar{\theta}^{i}=0 \text { in } \bar{\Omega} \backslash \Omega^{\#}, \forall i \in[1, l], \\
\frac{\partial \bar{\theta}^{i}}{\partial n}=0 \text { on } \Gamma, \forall i \in[1, l],
\end{gathered}
$$

and, if

$$
\bar{\theta}\left(x^{\prime}, t\right)=\sum_{i=1}^{l} h_{i}(t) \bar{\theta}^{i}\left(x^{\prime}\right), \forall\left(x^{\prime}, t\right) \in \bar{\Omega} \times[0,1]
$$

then

$$
\phi^{\nabla \bar{\theta}}(x, t, 0)=\bar{F}(t), \forall t \in[0,1] .
$$

Let $\gamma$ be a closed Jordan curve of class $C^{\infty}$ and let $r>0$ be such that, if we denote by $\Omega^{\gamma}$ the bounded connected component of $\mathbb{R}^{2} \backslash \gamma$,

$$
\begin{gathered}
\gamma \cup \Omega^{\gamma} \subset \Omega, \\
\phi^{\nabla \bar{\theta}}(B(x, r), t, 0) \subset \Omega^{\gamma}, \forall t \in[0,1], \\
\phi^{\nabla \bar{\theta}}(B(x, r), 1,0) \subset \Omega^{\#} \\
\Omega^{\#} \not \subset \gamma \cup \Omega^{\gamma} .
\end{gathered}
$$

The existence of such $\gamma$ and $r$ follows easily from (A.77), (A.78), (A.79), (A.82), and (A.87). By (A.91) there exists a nonempty open ball $\Omega_{1}^{\#}$ such that

$$
\begin{gathered}
\Omega_{1}^{\#} \subset \Omega^{\#}, \\
\Omega_{1}^{\#} \cap\left(\gamma \cup \Omega^{\gamma}\right)=\emptyset .
\end{gathered}
$$

Let us assume, for the moment being, that the following lemma holds Esaim: CoCV, May 1996, VOL. 1, PP. 35-75. 
Lemma A.3. For any $u \in C^{\infty}(\gamma ; \mathbb{R})$, for any $v \in C^{\infty}(\Gamma ; \mathbb{R})$, and for any $\nu>0$, there exist $\theta \in C^{\infty}(\bar{\Omega} ; \mathbb{R})$ such that

$$
\begin{gathered}
\Delta \theta=0 \text { in } \Omega \backslash \Omega_{1}^{\#}, \\
\frac{\partial \theta}{\partial n}=0 \text { on } \Gamma, \\
|\theta|_{\gamma}-\left.u\right|_{C^{0}(\gamma)}<\nu, \\
\left|\frac{d}{d \tau}\left(\left.\theta\right|_{\Gamma}\right)-\frac{d v}{d \tau}\right|_{C^{1}(\Gamma)}<\nu
\end{gathered}
$$

Let $\varepsilon>0$ be fixed. Let $\nu>0$ and let $i \in[1, l]$; by Lemma A.3, applied with $u=0$ and $v=\left.\bar{\theta}^{i}\right|_{\Gamma}$, there exists $\theta^{i} \in C^{\infty}(\bar{\Omega} ; \mathbb{R})$ such that

$$
\begin{gathered}
\Delta \theta^{i}=0 \text { in } \Omega \backslash \Omega_{1}^{\#}, \\
\frac{\partial \theta^{i}}{\partial n}=0 \text { on } \Gamma, \\
\left.\left|\theta^{i}\right|_{\gamma}\right|_{C^{\circ}(\gamma)}<\nu, \\
\left|\frac{d}{d \tau}\left(\left.\theta^{i}\right|_{\Gamma}\right)-\frac{d}{d \tau}\left(\left.\bar{\theta}^{i}\right|_{\Gamma}\right)\right|_{C^{1}(\Gamma)}<\nu,
\end{gathered}
$$

Let $\theta^{\varepsilon, x}:=\theta \in C^{\infty}(\bar{\Omega} \times[0,1] ; \mathbb{R})$ be defined by

$$
\theta\left(x^{\prime}, t\right)=\sum_{i=1}^{l} h_{i}(t)\left(\bar{\theta}^{i}-\theta^{i}\right)\left(x^{\prime}\right), \forall\left(x^{\prime}, t\right) \in \bar{\Omega} \times[0,1] .
$$

and take $\theta^{\varepsilon, x}=\theta$, with $\nu$ small enough. From (A.83) and (A.98), we get (A.2). From (A.84), (A.92), (A.94), and (A.98), we get (A.3). From (A.85), (A.95), and (A.98), we get (A.4). From (A.97) and (A.98), we get (A.5) if $\nu$ is small enough. Let us check (A.6) for $\nu$ small enough. Let $K_{1}$ be compact subset of $\Omega^{\gamma}$ such that

$$
\phi^{\nabla \bar{\theta}}(B(x, r), t, 0) \subset \text { interior of } K_{1}, \forall t \in[0,1] .
$$

The existence of such a compact subset follows from (A.89) (recall that $B(x, r)$ is a closed ball.) By (A.86), (A.93), (A.94), (A.96), and (A.98), there exists a constant $C$ which does not depend on $\nu$ such that

$$
|\nabla \theta-\nabla \bar{\theta}|_{L^{\infty}\left(K_{1} \times[0,1]\right)}<C \nu
$$

which, with (A.90) and (A.99), implies (A.6) if $\nu$ is small enough.

Finally we prove Lemma A.3. Note that this lemma is a controllability result. We proceed as J.-L. Lions in [19, Chapitre 2, Section 5.3] where a related result is proved. Assume that Lemma A.3 is false. Then there exist a bounded measure $M$ on $\bar{\Omega}$ and a distribution $w \in \mathcal{D}^{\prime}(\Gamma)$ on $\Gamma$ of order 1 such that

$$
\begin{aligned}
& \text { Support } M \subset \gamma, \\
& \left(M, \frac{\partial w}{\partial \tau}\right) \neq(0,0),
\end{aligned}
$$




$$
<w, \frac{\partial \varphi}{\partial \tau}>_{\mathcal{D}^{\prime}(\Gamma), \mathcal{D}(\Gamma)}+\int_{\bar{\Omega}} \varphi d M=0,
$$

for all $\varphi \in C^{\infty}(\bar{\Omega} ; \mathbb{R})$ such that

$$
\begin{gathered}
\Delta \varphi=0 \text { in } \bar{\Omega} \backslash \Omega_{1}^{\#}, \\
\frac{\partial \varphi}{\partial n}=0 \text { on } \Gamma .
\end{gathered}
$$

Let

$$
s \in(0,1 / 2) .
$$

Then (see, for example, [22, Chapitre 1]), one easily verifies that

$$
\begin{aligned}
& \frac{\partial w}{\partial \tau} \in H^{-\frac{5}{2}-s}(\Gamma), \\
& M \in H^{-1-s}(\Omega) .
\end{aligned}
$$

Using [22, Chapitre 2, Théorème 6.6] as well as (A.100), (A.103), (A.104), and (A.105), we get the existence of $\psi \in H^{-1-s}(\Omega)$ such that, with $\bar{M}=$ $\int_{\Omega} d M$

$$
\begin{gathered}
\Delta \psi=M-\bar{M}, \\
\frac{\partial \psi}{\partial n}=\frac{\partial w}{\partial \tau} .
\end{gathered}
$$

Let us notice that, in our situation, the meaning of (A.106)-(A.107) is (see $[22$, Chapitre $2,(6.25)]$ )

$$
\begin{aligned}
<\psi, \Delta k>_{H^{-1-s}(\Omega), H_{0}^{1+s}(\Omega)}=<M, & >_{\left(H^{3+s}(\Omega)\right)^{\prime}, H^{3+s}(\Omega)}-\bar{M} \int_{\Omega} k \\
& -<\frac{\partial w}{\partial \tau}, k>_{H^{-\frac{5}{2}-s}(\Gamma), H^{\frac{5}{2}+s}(\Gamma)}
\end{aligned}
$$

for any $k \in H^{3+s}(\Omega)$ such that

$$
\begin{aligned}
& \frac{\partial k}{\partial n}=0 \text { on } \Gamma, \\
& \Delta k=0 \text { on } \Gamma .
\end{aligned}
$$

In (A.108), $\left.H^{3+s}(\Omega)\right)^{\prime}$ denotes, as usual, the dual space of $H^{3+s}(\Omega)$. In particular, taking $k=\varphi$ in (A.108), we get, using (A.102),

$$
<\psi, \Delta \varphi>_{H^{-1-s}(\Omega), H_{0}^{1+s}(\Omega)}=-\bar{M} \int_{\Omega} \varphi .
$$

But, for any $f \in C^{\infty}(\bar{\Omega} ; \mathbb{R})$ such that

$$
\int_{\Omega} f=0
$$

and

$$
\text { Support } f \subset \Omega_{1}^{\#},
$$

there exists $\varphi \in C^{\infty}(\bar{\Omega} ; \mathbb{R})$ such that

$$
\Delta \varphi=f \text { in } \bar{\Omega},
$$




$$
\begin{gathered}
\int_{\Omega} \varphi=0 \\
\frac{\partial \varphi}{\partial n}=0 \text { on } \Gamma .
\end{gathered}
$$

Hence, from (A.111), we get the existence of a real number $C$ such that

$$
\psi=C \text { on } \Omega_{1}^{\#} \text {. }
$$

By (A.100), (A.106), and (A.112), we have

$$
\begin{gathered}
\bar{M}=0, \\
\Delta \psi=0 \text { in } \mathcal{D}^{\prime}(\Omega \backslash \gamma) .
\end{gathered}
$$

From (A.93), (A.112), and (A.114), we get

$$
\psi=C \text { in } \Omega \backslash\left(\gamma \cup \Omega^{\gamma}\right) .
$$

But, from (A.105) and (A.106), we get

$$
\psi \in H_{\text {loc }}^{1-s}(\Omega)
$$

which, with (A.103), (A.114), (A.116), and a classical trace theorem - see e.g. [22, Chapitre 1 , Théorème 8.3$]$-, implies that

$$
\psi=C \text { in } \Omega \text {. }
$$

From (A.106), (A.113), and (A.117), we get

$$
M=0 \text {. }
$$

Using (A.108), (A.117), and (A.118), we get

$$
<\frac{\partial w}{\partial \tau}, k>_{H^{-\frac{5}{2}-s}(\Gamma), H^{\frac{5}{2}+s}(\Gamma)}=0
$$

for all $k \in H^{3+s}(\Omega)$ such that (A.109) and (A.110) hold. But, for any $g \in C^{\infty}(\Gamma)$, there exists $k \in C^{\infty}(\bar{\Omega})$ such that (A.109) and (A.110) hold. So (A.119) implies that

$$
\frac{\partial w}{\partial \tau}=0
$$

which, with (A.101) and (A.118), gives a contradiction.

REMARK A.4. If one does not require (A.5), Lemma A.1 is already proved in [8]. Note that the method we have used in our proof of Lemma A.1 works also in higher dimension; this is not the case for the method used in [8].

\section{Appendix B. Proof of Lemma 2.2}

Let us denote by $\eta$ a positive real number such that, for any $x$ in $\bar{\Omega}$ with $d(x)<\eta$, there exists a unique $P(x)$ in $\partial \Omega$ such that

$$
d(x)=|x-P(x)|
$$

and such that $d$ is of class $C^{\infty}$ on $\{x \in \bar{\Omega} ; d(x)<\eta\}$. Let $h$ be a function in $C^{\infty}([0,+\infty) ;[0,2 / 3])$ such that

$$
\begin{gathered}
h^{\prime} \geqslant 0 \text { in }[0,+\infty), \\
h(s)=s, \forall s \in[0,1 / 2], \\
h(s)=1, \forall s \in[2 / 3,+\infty),
\end{gathered}
$$


and let $g: \bar{\Omega} \rightarrow[0,+\infty)$ be defined by

$$
g(x)=h^{2}\left(\frac{d(x)}{\eta}\right)
$$

Then, $g$ is of class $C^{\infty}$ and, for some positive real number $C_{1}$,

$$
\begin{gathered}
|\nabla g| \leqslant C_{1} d \text { in } \bar{\Omega}, \\
C_{1}^{-1} d^{2} \leqslant g \leqslant C_{1} d^{2} \text { in } \bar{\Omega} .
\end{gathered}
$$

Note that

$$
\Delta g=\frac{2}{\eta^{2}} \text { on } \Gamma
$$

and so, by (B.4), there exists a positive real number $C_{2}$ such that

$$
\Delta g \geqslant-C_{2} g \text { in } \bar{\Omega} \text {. }
$$

For a positive real number $C$, for $t^{*} \in\left(0, C^{-1}\right]$, for $\psi \in C^{\infty}\left(\bar{\Omega} \times\left[0, t^{*} ; \mathbb{R}\right)\right.$, for $X \in C^{\infty}\left(\bar{\Omega} \times\left[0, t^{*}\right] ; \mathbb{R}^{2}\right)$, and $Y \in C^{\infty}\left(\bar{\Omega} \times\left[0, t^{*}\right] ; \mathbb{R}^{2}\right)$ satisfying (2.71) to $(2.75)$, let $\bar{\psi} \in C^{\infty}\left(\bar{\Omega} \times\left(0, t^{*}\right] ;[0,+\infty)\right)$ be defined by

$$
\begin{aligned}
\bar{\psi}(\cdot, t)=\left(\exp C t|X|_{L^{\infty}}^{2}\right)\left(\exp C t^{\frac{3 \beta}{2}}\left|\nabla^{2} Y\right|_{L^{\infty}}\right) \\
\exp -\left(\frac{1}{(C t)^{\alpha}} f\left(\frac{g}{(C t)^{\beta}}\right)\right), \forall t \in\left(0, t^{*}\right],
\end{aligned}
$$

where, for simplicity, we have written $|X|_{L^{\infty}}$ for $|X|_{L^{\infty}\left(\Omega \times\left[0, t^{*}\right]\right)}$ and $\left|\nabla^{2} Y\right|_{L^{\infty}}$ for $\left|\nabla^{2} Y\right|_{L^{\infty}(\Omega \times[0, T])}$. One has (recall $(2.70)$ )

$$
\begin{gathered}
\frac{\partial \bar{\psi}}{\partial t}=\left(C|X|_{L^{\infty}}^{2}+\frac{3 \beta}{2} C t^{\frac{3 \beta}{2}-1}\left|\nabla^{2} Y\right|_{L^{\infty}}\right. \\
\left.+\frac{\alpha}{C^{\alpha} t^{1+\alpha}} f\left(\frac{g}{(C t)^{\beta}}\right)+\frac{\beta g}{C t^{2}} f^{\prime}\left(\frac{g}{(C t)^{\beta}}\right)\right) \bar{\psi} \\
\frac{\partial \bar{\psi}}{\partial x_{i}}=-\frac{1}{C t}\left(\frac{\partial g}{\partial x_{i}}\right) f^{\prime}\left(\frac{g}{(C t)^{\beta}}\right) \bar{\psi} \\
\Delta \bar{\psi}=\left(-\frac{1}{C t}(\Delta g) f^{\prime}\left(\frac{g}{(C t)^{\beta}}\right)-\frac{1}{(C t)^{1+\beta}}|\nabla g|^{2} f^{\prime \prime}\left(\frac{g}{(C t)^{\beta}}\right)\right. \\
\left.+\frac{1}{C^{2} t^{2}}|\nabla g|^{2}\left(f^{\prime}\right)^{2}\left(\frac{g}{(C t)^{\beta}}\right)\right) \bar{\psi}
\end{gathered}
$$

Let us denote by $C_{j}, j \geqslant 3$, various positive constants which do not depend on $C, t^{*}, \psi, X, Y, x$, and $t$. By (2.68), (B.3), (B.4), and (B.7) one has, for some $C_{3}$ in $(0,+\infty)$,

$$
\begin{aligned}
|(X \cdot \nabla) \bar{\psi}| & \leqslant \frac{1}{C t}|X|_{L^{\infty}}|\nabla g| f^{\prime}\left(\frac{g}{(C t)^{\beta}}\right) \bar{\psi} \\
& \leqslant|X|_{L^{\infty}}^{2} \bar{\psi}+\frac{|\nabla g|^{2}}{4 C^{2} t^{2}} f^{\prime}\left(\frac{g}{(C t)^{\beta}}\right) \bar{\psi} \\
& \leqslant|X|_{L^{\infty}}^{2} \bar{\psi}+C_{3} \frac{g}{C^{2} t^{2}} f^{\prime}\left(\frac{g}{(C t)^{\beta}}\right) \bar{\psi} .
\end{aligned}
$$


By (B.1), (B.2), and (B.7), one has

$$
\frac{\partial \bar{\psi}}{\partial x_{i}}(x, t)=0 \text { if } d(x) \geqslant 2 \eta / 3 .
$$

Let us now assume that $d(x) \leqslant 2 \eta / 3$; then we have

$$
\begin{aligned}
\sum_{i=1}^{2} Y^{i}(x, t) \frac{\partial \bar{\psi}}{\partial x_{i}}(x, t)=\sum_{i=1}^{2} & Y^{i}(P(x), t) \frac{\partial \bar{\psi}}{\partial x_{i}}(x, t) \\
& +\sum_{i=1}^{2}\left(Y^{i}(x, t)-Y^{i}(P(x), t)\right) \frac{\partial \bar{\psi}}{\partial x_{i}}(x, t) .
\end{aligned}
$$

Note that, by (2.75), (B.2), and (B.7), the first sum of the right hand side of (B.11) vanishes. Therefore, since $x-P(x)$ is colinear to $n(P x),(\mathrm{B} .11)$, together with (2.74), (B.3), (B.4), (B.7), and (B.10), implies that, for some $C_{4}$ in $(0,+\infty)$

$$
|(Y \cdot \nabla) \bar{\psi}| \leqslant C_{4}\left(\frac{g}{C^{2} t^{2}}+\frac{g^{3 / 2}}{C t}\left|\nabla^{2} Y\right|_{L^{\infty}}\right) f^{\prime}\left(\frac{g}{(C t)^{\beta}}\right) \bar{\psi} .
$$

From (2.67), (2.68), (2.69), (B.3), (B.4), (B.5), (B.6), (B.8), (B.9), and (B.12), one easily verifies that, for some $C_{5}$ in $(0,+\infty)$, one has, for all $C$ in $\left[C_{5},+\infty\right)$ and for all $t^{*} \in\left(0, C^{-1}\right]$,

$$
\frac{\partial \bar{\psi}}{\partial t}-\Delta \bar{\psi}+((X+Y) \cdot \nabla) \bar{\psi} \geqslant 0 \text { in } \bar{\Omega} \times\left(0, t^{*}\right]
$$

Since

$$
\begin{aligned}
& \bar{\psi} \geqslant 1 \text { on } \Gamma \times\left(0, t^{*}\right], \\
& \bar{\psi} \geqslant 0 \text { in } \bar{\Omega} \times\left(0, t^{*}\right],
\end{aligned}
$$

we have, using (2.71), (2.72), (2.73), (B.13), and the maximum principle,

$$
\psi \leqslant \bar{\psi} \text { in } \bar{\Omega} \times\left(0, t^{*}\right]
$$

which, with (2.67), (2.68), (2.69), and (B.4), ends the proof of Lemma 2.2.

\section{Appendix C. Proof of Lemma 2.3}

Clearly, by linearity, we may assume that

$$
B^{*}=1 \text {. }
$$

Let us also point out that, without loss of generality, we may assume that

$$
f(s)=s, \forall s \in[0,+\infty) .
$$

Indeed, this follows easily from the fact that, by $(2.70),(2.86)$ and $(2.87)$,

$$
0 \leqslant\left(\exp -A^{\alpha} f\left(A^{\beta} s^{2}\right)\right)-\exp -A s^{2} \leqslant \exp -A^{\alpha} .
$$

From now on we assume that (C.1) and (C.2) hold; so (2.88) reads

$$
|\Delta \psi| \leqslant B+\exp -A d^{2} \text { in } \bar{\Omega} .
$$

Then let us point out that, again without loss of generality, we may assume that

$$
B=0 \text {. }
$$


Indeed, let $h: \bar{\Omega} \rightarrow \mathbb{R}$ be defined by

$$
\begin{gathered}
\Delta h=\operatorname{Min}\left\{B, \Delta \psi+\exp -A d^{2}\right\}, \\
h=0 \text { on } \Gamma .
\end{gathered}
$$

Then, from (C.10), we get

$$
\begin{gathered}
|\Delta h| \leqslant B, \\
|\Delta(\psi-h)| \leqslant \exp -A d^{2} .
\end{gathered}
$$

From (C.6) and (C.7) we get, for some $C_{0}>0$ depending only on $\Omega$,

$$
|h|+|\nabla h| \leqslant C_{0} B \text { in } \bar{\Omega} \text {. }
$$

Hence, replacing if necessary $\psi$ by $\psi-h$, we may assume (C.4) which with (C.3) gives

$$
|\Delta \psi| \leqslant \exp -A d^{2} \text { in } \bar{\Omega} .
$$

Let $G:(\bar{x}, x) \rightarrow G(\bar{x}, x)$ be the Green function of the domain $\Omega ; G$ is a function of class $C^{\infty}$ on $\left\{(\bar{x}, x) \in \bar{\Omega}^{2} ; x \neq \bar{x}\right\}$ which satisfies

$$
\begin{gathered}
\Delta_{x} G(\bar{x}, \cdot)=\delta_{\bar{x}}, \forall \bar{x} \in \bar{\Omega}, \\
G(\bar{x}, \cdot)=0 \text { on } \Gamma \backslash\{\bar{x}\}, \forall \bar{x} \in \bar{\Omega} .
\end{gathered}
$$

By (2.89), (C.11), and (C.12) one has, for all $\bar{x}$ in $\bar{\Omega}$,

$$
\psi(\bar{x})=\int_{\Omega} G(\bar{x}, x) \Delta \psi(x) .
$$

We differentiate (C.13) with respect to $\bar{x}$ and get

$$
\nabla \psi(\bar{x})=\int_{\Omega} \nabla_{\bar{x}} G(\bar{x}, x) \Delta \psi(x) .
$$

Let $\eta<\bar{\eta}$ where $\bar{\eta}>0$ satisfies property $(\mathcal{P})$ on page 55 . Let us denote by $C_{i}, i \geqslant 1$, various positive constants which may depend on $\Omega$ and $\eta$ but are independent of $\bar{x}, x, \psi, \mu \in(0,1]$ and of $A \geqslant 1$. For example, for some constant $C_{1}>0$, one has the following classical estimates

$$
\begin{gathered}
\left|\nabla_{\bar{x}} G(\bar{x}, x)\right| \leqslant \frac{C_{1}}{|x-\bar{x}|}, \forall(x, \bar{x}) \in \bar{\Omega}^{2} \text { with } x \neq \bar{x}, \\
\left|\nabla_{\bar{x}} G(\bar{x}, x)\right| \leqslant C_{1} d(x), \forall(x, \bar{x}) \in \bar{\Omega}^{2} \text { with } d(x) \leqslant \frac{\eta}{4} \text { and } d(\bar{x}) \geqslant \frac{\eta}{2} . \text { (C.16) }
\end{gathered}
$$

Let us first estimate $|\nabla \psi(\bar{x})|$ when

$$
d(\bar{x}) \geqslant \frac{\eta}{2}
$$

Let, for $\nu>0$,

$$
\Omega_{\nu}=\{x \in \bar{\Omega} ; d(x)<\nu\} .
$$

By (C.10), (C.14), (C.15), (C.16), and (C.17), one has, for some $C_{2} \in$ $(0,+\infty)$

$$
|\nabla \psi(\bar{x})| \leqslant C_{2}\left(\int_{\Omega_{\eta / 4}} d \exp -A d^{2}+\int_{\Omega \backslash \Omega_{\eta / 4}} \exp -A\left(\frac{\eta}{4}\right)^{2}\right) .
$$


But, for some $C_{3}$ in $(0,+\infty)$,

$$
\int_{\Omega_{\eta / 4}} d \exp -A d^{2} \leqslant \frac{C_{3}}{A}
$$

which, with (C.18), implies that, for some $C_{4}$ in $(0,+\infty)$,

$$
|\nabla \psi(\bar{x})| \leqslant \frac{C_{4}}{A}, \forall \bar{x} \in \Omega \backslash \Omega_{\eta / 2} .
$$

We now turn to the case where

$$
d(\bar{x})<\frac{\eta}{2} .
$$

Let, for $\nu>0$ and $i \in[0, g]$,

$$
\Omega_{\nu}^{i}=\left\{x \in \bar{\Omega} ; \operatorname{dist}\left(x, \Gamma_{i}\right)<\nu\right\} .
$$

One has

$$
\Omega_{\nu}=\cup_{i=0}^{g} \Omega_{\nu}^{i}
$$

and, by (A.7), if $\nu \in[0, \bar{\eta})$, and if $(i, j) \in[0, g]^{2}$ is such that $i \neq j$,

$$
\left|x_{1}-x_{2}\right| \geqslant 2(\bar{\eta}-\nu), \forall\left(x_{1}, x_{2}\right) \in \Omega_{\nu}^{i} \times \Omega_{\nu}^{j} .
$$

Without loss of generality, we may assume that

$$
\bar{x} \in \Omega_{\eta / 2}^{0} .
$$

Then, proceeding as above and using (C.10), (C.14), (C.15), (C.16), and (C.20), we get that, for some $C_{5}$ in $(0,+\infty)$,

$$
|\nabla \psi(\bar{x})| \leqslant C_{5}\left(\frac{1}{A}+f(\bar{x})\right)
$$

with

$$
f(\bar{x})=\int_{\Omega_{\eta}^{0}}\left|\nabla_{\bar{x}} G(\bar{x}, \cdot)\right| \exp -A d^{2} .
$$

Let $L$ be the length of $\Gamma_{0}$ and let us fix a point on $\Gamma^{0}$; let us parametrise a point in $\Gamma_{0}$ by the arclength $s \in[0, L)$ from this fixed point $-\Gamma_{0}$ is equipped with the orientation given by the vector field $\tau$-. One can define a parametrisation of $\Omega_{\eta}^{0}$ by assiocating to $s$ in $[0, L)$ and $\rho$ in $[0, \eta)$ the unique point $x$ in $\Omega_{\eta}^{0}$ such that $d(x)=\eta$ and such that $P(x)$ is the point of $\Gamma_{0}$ corresponding to the parameter $s \in[0, L)$. Let us denote by $\bar{s}$ and $\bar{\rho}$ the parameters corresponding to $\bar{x}$. Let us assume for the moment being that, for some positive constant $C_{6}$,

$$
\left|\nabla_{\bar{x}} G(\bar{x}, x)\right| \leqslant \frac{C_{6} \rho\left(\bar{\rho}|s-\bar{s}|+(s-\bar{s})^{2}+\left|\rho^{2}-\bar{\rho}^{2}\right|\right)}{\left((s-\bar{s})^{2}+(\rho+\bar{\rho})^{2}\right)\left((s-\bar{s})^{2}+(\rho-\bar{\rho})^{2}\right)} .
$$

From (C.22) and (C.23) we get that, for some $C_{7}$ in $(0,+\infty)$,

$$
\begin{aligned}
f(\bar{x}) \leqslant C_{7} \int_{0}^{\eta} \rho\left(\bar{\rho} g_{1}(\bar{x}, \rho)+g_{2}(\bar{x}, \rho)\right. & \\
& \left.+\left|\rho^{2}-\bar{\rho}^{2}\right| g_{3}(\bar{x}, \rho)\right) \exp -A \rho^{2} d \rho
\end{aligned}
$$

with

$$
g_{1}(\bar{x}, \rho)=\int_{0}^{L} \frac{|s-\bar{s}|}{\left((s-\bar{s})^{2}+(\rho+\bar{\rho})^{2}\right)\left((s-\bar{s})^{2}+(\rho-\bar{\rho})^{2}\right)} d s,
$$




$$
\begin{aligned}
& g_{2}(\bar{x}, \rho)=\int_{0}^{L} \frac{(s-\bar{s})^{2}}{\left((s-\bar{s})^{2}+(\rho+\bar{\rho})^{2}\right)\left((s-\bar{s})^{2}+(\rho-\bar{\rho})^{2}\right)} d s \\
& g_{3}(\bar{x}, \rho)=\int_{0}^{L} \frac{1}{\left((s-\bar{s})^{2}+(\rho+\bar{\rho})^{2}\right)\left((s-\bar{s})^{2}+(\rho-\bar{\rho})^{2}\right)} d s .
\end{aligned}
$$

One has

$$
\begin{aligned}
g_{1}(\bar{x}, \rho) & \leqslant 2 \int_{0}^{L} \frac{s}{\left(s^{2}+(\rho+\bar{\rho})^{2}\right)\left(s^{2}+(\rho-\bar{\rho})^{2}\right)} d s \\
& \leqslant \int_{0}^{+\infty} \frac{1}{\left(u+(\rho+\bar{\rho})^{2}\right)\left(u+(\rho-\bar{\rho})^{2}\right)} d u \\
& \leqslant \frac{1}{2 \rho \bar{\rho}} \ln \frac{\rho+\bar{\rho}}{|\rho-\bar{\rho}|},
\end{aligned}
$$

which implies that, for some $C_{8}$ in $(0,+\infty)$,

$$
\begin{aligned}
\int_{0}^{\eta} \rho \bar{\rho} g_{1}(\bar{x}, \rho) \exp -A \rho^{2} d \rho & \leqslant \frac{1}{2} \int_{0}^{\eta} \ln \frac{\rho+\bar{\rho}}{|\rho-\bar{\rho}|} \exp -A \rho^{2} d \rho \\
& \leqslant \frac{1}{2 \sqrt{A}} \int_{0}^{+\infty} \ln \frac{u+\bar{\rho} \sqrt{A}}{|u-\bar{\rho} \sqrt{A}|} \exp -u^{2} d u \\
& \leqslant \frac{C_{8}}{\sqrt{A}}
\end{aligned}
$$

Clearly

$$
\begin{aligned}
g_{2}(\bar{x}, \rho) & \leqslant 2 \int_{0}^{+\infty} \frac{1}{s^{2}+(\rho+\bar{\rho})^{2}} d s=\frac{\pi}{\rho+\bar{\rho}} \\
& \leqslant \frac{\pi}{\rho}
\end{aligned}
$$

which implies that

$$
\int_{0}^{\eta} \rho g_{2}(\bar{x}, \rho) \exp -A \rho^{2} d \rho \leqslant \frac{\pi}{\sqrt{A}} \int_{0}^{+\infty} \exp -u^{2} d u
$$

Similarly, one has

$$
\begin{aligned}
g_{3}(\bar{x}, \rho) & \leqslant 2 \int_{0}^{+\infty} \frac{1}{\left(s^{2}+(\rho+\bar{\rho})^{2}\right)\left(s^{2}+(\rho-\bar{\rho})^{2}\right)} d s \\
& \leqslant \frac{2}{(\rho+\bar{\rho})^{2}} \int_{0}^{+\infty} \frac{1}{s^{2}+(\rho-\bar{\rho})^{2}} d s=\frac{\pi}{(\rho+\bar{\rho})^{2}|\rho-\bar{\rho}|} \\
& \leqslant \frac{\pi}{\rho\left|\rho^{2}-\bar{\rho}^{2}\right|}
\end{aligned}
$$

which implies that

$$
\int_{0}^{\eta} \rho\left|\rho^{2}-\bar{\rho}^{2}\right| g_{3}(\bar{x}, \rho) \exp -A \rho^{2} d \rho \leqslant \frac{\pi}{\sqrt{A}} \int_{0}^{+\infty} \exp -u^{2} d u .
$$

From (C.21), (C.24), (C.25), (C.26), (C.27), (C.29), (C.31), and (C.33), we get that, for some $C_{9}$ in $(0,+\infty)$,

$$
|\nabla \psi(\bar{x})| \leqslant \frac{C_{9}}{\sqrt{A}}, \forall x \in \Omega_{\eta / 2}^{0},
$$

which, with (C.19), ends the proof of (2.90).

Esaim: CoCv, May 1996, VOL. 1, PP. 35-75. 
We now prove (2.91). Let us first point out that, from (C.19), we get the existence of $C_{10}$ in $(0,+\infty)$ such that

$$
|\nabla \psi|_{L^{1}\left(\Omega \backslash \Omega_{\eta / 2}\right)} \leqslant \frac{C_{10}}{A}
$$

Define the integral

$$
I_{1}=\int_{\Omega_{\eta / 2}^{0}} \int_{0}^{\eta} d(\bar{x})^{\mu} \rho \bar{\rho} g_{1}(\bar{x}, \rho) \exp -A \rho^{2} .
$$

From (C.28), we get, for some $C_{11}, C_{12}, C_{13}$, and $C_{14}$ in $(0,+\infty)$,

$$
\begin{aligned}
I_{1} & \leqslant C_{11} \int_{0}^{\eta / 2} \int_{0}^{\eta} \bar{\rho}^{\mu} \ln \frac{\rho+\bar{\rho}}{|\rho-\bar{\rho}|} \exp -A \rho^{2} d \rho \\
& \leqslant \frac{C_{11}}{A^{1+\frac{\mu}{2}}} \int_{0}^{\frac{\eta \sqrt{A}}{2}} \int_{0}^{+\infty} u^{\mu} \ln \frac{u+v}{|u-v|} \exp -v^{2} d u d v \\
& \leqslant \frac{C_{11}}{A^{1+\frac{\mu}{2}}}\left(\iint_{0<u<2 v}+\iint_{2 v<u<\frac{\eta}{2} \sqrt{A}} u^{\mu} \ln \frac{u+v}{|u-v|} \exp -v^{2}\right) \\
& \leqslant \frac{C_{11}}{A^{1+\frac{\mu}{2}}}\left(C_{12}+\frac{C_{13}}{\mu} A^{\frac{\mu}{2}}\right) \\
& \leqslant \frac{C_{14}}{\mu A}
\end{aligned}
$$

Let

$$
I_{2}=\int_{\Omega_{\eta / 2}^{0}} \int_{0}^{\eta} d(\bar{x})^{\mu} \rho g_{2}(\bar{x}, \rho) \exp -A \rho^{2} d \rho .
$$

From (C.30), we get, for some $C_{15}$ and $C_{16}$ in $(0,+\infty)$,

$$
\begin{aligned}
I_{2} & \leqslant C_{15} \int_{\Omega_{\eta / 2}^{0}} \int_{0}^{\eta} \bar{\rho}^{\mu} \frac{\rho}{\rho+\bar{\rho}} \exp -A \rho^{2} d \rho \\
& \leqslant \frac{C_{15}}{A^{1+\frac{\mu}{2}}} \int_{0}^{\frac{\eta \sqrt{A}}{2}} \int_{0}^{+\infty} u^{\mu} \frac{v}{u+v} \exp -A v^{2} d u d v \\
& \leqslant \frac{C_{15}}{A^{1+\frac{\mu}{2}}}\left(\int_{0}^{+\infty} v \exp -v^{2} d v\right)\left(\frac{1}{\mu}\left(\frac{\eta \sqrt{A}}{2}\right)^{\mu}\right) \\
& \leqslant \frac{C_{16}}{\mu A} .
\end{aligned}
$$

Let

$$
I_{3}=\int_{\Omega_{\eta / 2}^{0}} \int_{0}^{\eta} d(\bar{x})^{\mu} \rho\left|\rho^{2}-\bar{\rho}^{2}\right| g_{3}(\bar{x}, \rho) \exp -A \rho^{2} d \rho .
$$

From (C.32), we get, for some $C_{17}$ and $C_{18}$ in $(0,+\infty)$,

$$
\begin{aligned}
I_{3} & \leqslant C_{17} \int_{0}^{\eta / 2} \int_{0}^{\eta} \bar{\rho}^{\mu} \frac{\rho}{\rho+\bar{\rho}} \exp -A \rho^{2} d \rho \\
& \leqslant \frac{C_{18}}{\mu A} .
\end{aligned}
$$


From (C.21), (C.24), (C.35), (C.36), (C.37), (C.38), (C.39), and (C.40), we obtain, for some $C_{19}$ in $(0,+\infty)$,

$$
\int_{\Omega_{\eta / 2}^{0}} d^{\mu}|\nabla \psi| \leqslant \frac{C_{19}}{\mu A} .
$$

Of course, using the same method we can show that for some $C_{20}$ in $(0,+\infty)$

$$
\int_{\Omega_{\eta / 2}^{i}} d^{\mu}|\nabla \psi| \leqslant \frac{C_{20}}{\mu A}, \forall i \in[1, g],
$$

which, with (C.34) and (C.41), ends the proof of (2.91) if (C.23) holds.

Let us prove that (C.23) holds. We argue by contradiction and so assume that (C.23) does not hold. Then there exists a sequence $\left\{\left(\bar{x}_{k}, x_{k}\right) \in \Omega_{\eta}^{0} \times\right.$ $\left.\Omega_{\eta}^{0} ; k \in \mathbb{N}\right\}$ such that

$$
\begin{gathered}
\bar{x}_{k} \neq x_{k}, \forall k \in \mathbb{N} \\
\left|\nabla_{\bar{x}_{k}} G\left(\bar{x}_{k}, x_{k}\right)\right| \geqslant k J_{k}, \forall k \in \mathbb{N},
\end{gathered}
$$

with

$$
J_{k}=\rho_{k} \frac{\left.\bar{\rho}_{k}\left|s_{k}-\bar{s}_{k}\right|+\left|s_{k}-\bar{s}_{k}\right|^{2}+\left|\rho_{k}^{2}-\bar{\rho}_{k}^{2}\right|\right)}{\left(\left(s_{k}-\bar{s}_{k}\right)^{2}+\left(\rho_{k}+\bar{\rho}_{k}\right)^{2}\right)\left(\left(s_{k}-\bar{s}_{k}\right)^{2}+\left(\rho_{k}-\bar{\rho}_{k}\right)^{2}\right)} .
$$

One easily checks that

$$
\begin{gathered}
\left|x_{k}-\bar{x}_{k}\right| \rightarrow 0 \text { as } k \rightarrow+\infty, \\
\rho_{k}+\bar{\rho}_{k} \rightarrow 0 \text { as } k \rightarrow+\infty .
\end{gathered}
$$

Let, for $k \in \mathbb{N}$,

$$
\lambda_{k}=\frac{1}{\left|x_{k}-\bar{x}_{k}\right|}
$$

and let $\mathcal{R}_{k}$ be the rotation of $\mathbb{R}^{2}$ such that

$$
\mathcal{R}_{k}\left(P\left(x_{k}\right)-x_{k}\right) \in(-\infty, 0) \times \mathbb{R} .
$$

Let also

$$
\begin{gathered}
\bar{x}_{k}^{*}=\lambda_{k} \mathcal{R}_{k}\left(\bar{x}_{k}-x_{k}\right), \\
\Omega_{k}^{*}=\left\{\lambda_{k} \mathcal{R}_{k}\left(x-x_{k}\right) ; x \in \Omega\right\},
\end{gathered}
$$

and let $G_{k}^{*}:\left(a_{1}, a_{2}\right) \rightarrow G_{k}^{*}\left(a_{1}, a_{2}\right)$ be the Green function of the open set $\Omega_{k}^{*}$. One has

$$
\nabla_{a_{1}} G_{k}^{*}\left(\bar{x}_{k}^{*}, 0\right)=\frac{1}{\lambda_{k}} \nabla_{\bar{x}_{k}} G\left(\bar{x}_{k}, x_{k}\right) .
$$

Note that, by (C.44) and (C.45),

$$
\left|\bar{x}_{k}^{*}\right|=1, \forall k \in \mathbb{N} \text {. }
$$

So, extracting if necessary a subsequence, we may assume without loss of generality that $\left(\bar{x}_{k}^{*} ; k \in \mathbb{N}\right)$ is convergent in $\mathbb{R}^{2}$ :

$$
\bar{x}_{k}^{*} \rightarrow \bar{x}^{*} \text { as } k \rightarrow+\infty, \text { with }\left|\bar{x}^{*}\right|=1 .
$$

Extracting again subsequences, we may assume without loss of generality that either

$$
\lambda_{k} \rho_{k} \rightarrow+\infty \text { as } k \rightarrow+\infty
$$


or

$$
\lambda_{k} \rho_{k} \rightarrow c \in[0,+\infty) \text { as } k \rightarrow+\infty .
$$

Let us first study the case where (C.49) holds. In this case, if we denote by $G_{\mathbb{R}^{2}}$ the Green function of $\mathbb{R}^{2}$ - i.e. $G\left(a_{1}, a_{2}\right)=(1 / 2 \pi) \ln \left|a_{1}-a_{2}\right|$ - we have using (C.48)

$$
\left|\nabla_{a_{1}} G_{k}^{*}\left(\bar{x}_{k}^{*}, 0\right)\right| \rightarrow\left|\nabla_{a_{1}} G_{\mathbb{R}^{2}}\left(\bar{x}^{*}, 0\right)\right|=\frac{1}{2 \pi} \frac{1}{\left|\bar{x}^{*}\right|}
$$

as $k \rightarrow+\infty$. But one easily checks that

$$
\liminf _{k \rightarrow+\infty} \frac{J_{k}}{\lambda_{k}} \geqslant \frac{1}{4}>0
$$

which gives a contradiction with (C.42), (C.47), and (C.51).

Let us finally study the case where (C.50) holds. Let

$$
\Omega_{\infty}^{*}=(-c,+\infty) \times \mathbb{R}
$$

and, for $k \in[0,+\infty]$, let $J_{k}^{*}:\left(\bar{\Omega}_{k}^{*} \times \bar{\Omega}_{k}^{*}\right) \backslash\left\{\left(a_{1}, a_{1}\right) ; a_{1} \in \mathbb{R}^{2}\right\} \rightarrow \mathbb{R}$ be defined by

$$
\begin{gathered}
J_{k}^{*}\left(a_{1}, a_{2}\right)=\frac{1}{\operatorname{dist}\left(a_{2}, \partial \Omega_{k}^{*}\right)}\left|\nabla_{a_{1}} G_{k}^{*}\left(a_{1}, a_{2}\right)\right| \text { if } a_{2} \notin \partial \Omega_{k}^{*}, \\
J_{k}^{*}\left(a_{1}, a_{2}\right)=\left|\nabla_{a_{1}}\left(n_{k}^{*}\left(a_{2}\right) \cdot \nabla_{a_{2}} G_{k}^{*}\left(a_{1}, a_{2}\right)\right)\right| \text { if } a_{2} \in \partial \Omega_{k}^{*},
\end{gathered}
$$

where $n_{k}^{*}$ denotes the outward unit normal vector field on $\partial \Omega_{k}^{*}$. Note that $J_{k}^{*}$ is continuous. One has, using in particular (C.48) and (C.50),

$$
J_{k}^{*}\left(\bar{x}_{k}^{*}, 0\right) \rightarrow J_{\infty}^{*}\left(\bar{x}^{*}, 0\right) \text { as } k \rightarrow+\infty .
$$

Note that -see (C.46) -

$$
\operatorname{dist}\left(0, \partial \Omega_{k}^{*}\right)=\lambda_{k} \rho_{k}, \forall k \in \mathbb{N} .
$$

Moreover, as one easily cheks,

$$
\liminf _{k \rightarrow+\infty} \frac{J_{k}}{\lambda_{k}^{2} \rho_{k}}>0
$$

which gives a contradiction with (C.42), (C.47), (C.52), (C.53), and (C.54) and ends the proof of (C.23).

Finally we prove $(2.92)$. We could proceed as in the proof of $(2.90)$; but it is simpler to take advantage of the maximum principle and the conformal mapping theorem in order to shorten the computations. For $j \in[0, g]$, let $\bar{\psi}_{j} \in C^{\infty}(\bar{\Omega} ; \mathbb{R})$ be defined by

$$
\begin{gathered}
-\Delta \bar{\psi}_{j}=\exp -A d_{j}^{2} \text { in } \bar{\Omega}, \\
\bar{\psi}_{j}=0 \text { on } \Gamma,
\end{gathered}
$$

where $d_{j}=\operatorname{dist}\left(\cdot, \Gamma_{j}\right)$. By $(2.89),(\mathrm{C} .10),(\mathrm{C} .55)$, and (C.56),

$$
\begin{gathered}
|\Delta \psi| \leqslant-\Delta \sum_{j=0}^{g} \bar{\psi}_{j} \text { in } \bar{\Omega}, \\
\psi=\sum_{j=0}^{g} \bar{\psi}_{j} \text { on } \Gamma,
\end{gathered}
$$


which, with the maximum principle, imply that

$$
|\psi| \leqslant \sum_{j=0}^{g} \bar{\psi}_{j} \text { in } \bar{\Omega} .
$$

Let $j \in[0, g]$; performing, if necessary, an inversion we may assume that $\Omega$ is included in the bounded connected component of $\mathbb{R}^{2} \backslash \Gamma_{j}$ (note that if $h: \Omega^{\prime} \rightarrow \Omega$ is a conformal map, then $\Delta(\varphi \circ h)=(1 / 2)|\nabla h|^{2}((\Delta \varphi) \circ h)$.) Similarly, using the conformal mapping theorem, one sees that, without loss of generality, we may assume that $\Gamma_{j}$ is the unit circle. Then, let $B_{1}=\left\{x \in \mathbb{R}^{2} ;|x| \leqslant 1\right\}$ and let $\bar{\psi} \in C^{\infty}\left(B_{1} ; \mathbb{R}\right)$ be defined by

$$
\begin{gathered}
-\Delta \bar{\psi}=\exp -A\left(1-|x|^{2}\right) \text { in } B_{1}, \\
\bar{\psi}=0 \text { in } \partial B_{1} .
\end{gathered}
$$

By the maximum principle one has

$$
\bar{\psi}_{j} \leqslant \bar{\psi}_{\text {in }} B_{1} \text {. }
$$

But we have

$$
\bar{\psi}(x)=\int_{|x|}^{1} \frac{1}{u}\left(\int_{0}^{u} v \exp -A(1-v)^{2} d v\right) d u .
$$

So, for some $C_{21}$ in $(0,+\infty)$,

$$
\begin{aligned}
\bar{\psi}(x) & \leqslant \psi(0)=\int_{0}^{1}|\ln v| v \exp -A(1-v)^{2} d v \\
& \leqslant C_{21} \int_{0}^{1}(1-v) \exp -A(1-v)^{2} d v \\
& \leqslant \frac{C_{21}}{2 A}
\end{aligned}
$$

which, with (C.57) and (C.58), ends the proof of (2.92).

\section{REFERENCES}

[1] R.A. Adams: Sobolev spaces, Academic Press, San Diego, London, 1978.

[2] C. Bardos, F. Golse, and D. Levermore: Fluid dynamic limits of kinetic equations I: formal derivations, J. Statistical Physics, 63, 1991, 323-344.

[3] F. Coron: Derivation of slip boundary conditions for the Navier-Stokes system from the Boltzmann equation, J. Statistical Physics, 54, 1989, 829-857.

[4] J.-M. Coron: Global asymptotic stabilization for controllable systems without drift, Math. Control Signals Systems, 5, 1992, 295-312.

[5] J.-M. Coron: Stabilization of controllable systems, preprint, 1993, to appear in Nonholonomic geometry, A. Bellaïche and J.-J. Risler ed. , Progress in Math., Birkhäuser.

[6] J.-M. Coron: Relations entre commandabilité et stabilisations non linéaires, in Nonlinear partial differential equations and their applications, Collège de France seminars, Paris 1989-1991, Vol.11, H. Brezis and J.-L. Lions eds., Pitman Res. Notes Math. Ser., London, 299, 1994, 68-86.

[7] J.-M. Coron: Contrôlabilité exacte frontière de l'équation d'Euler des fluides parfaits incompressibles bidimensionnels, C. R. Acad. Sci. Paris, 317, 1993, 271-276.

[8] J.-M. Coron: On the controllability of 2-D incompressible perfect fluids, J. Math. Pures \&́ Appliquées, 75, 1996, 155-188.

[9] C. Fabre: Uniqueness result for Stokes equations and their consequences in linear and nonlinear control problems, in Contrôlabilité approchée des solutions de quelques équations d'évolution, Habilitation à diriger des recherches, Université Pierre et Marie Curie, January 1996.

Esaim: Cocv, May 1996, Vol. 1, PP. 35-75. 
[10] E. Fernández-Cara and M. González-Burgos: A result concerning approximate controllability for the Navier-Stokes Equations, SIAM J. Control, to appear.

[11] E. Fernández-Cara and J. Real: On a conjecture due to J.-L. Lions, Nonlinear Analysis, Theory, Methods \& Appl., 21, 1993, 835-847.

[12] A.V. Fursikov: Exact boundary zero controllability of three-dimensional NavierStokes equations, J. Dynamical Control \&3 Systems, 1, 1995, 325-350.

[13] A.V. Fursikov and O.Yu. Imanuvilov: On controllability of certain systems simulating a fluid flow, in Flow Control, IMA vol. in Math. and its Appl. , M.D. Gunzburger ed., Springer Verlag, New York, 68, 1995, 149-184.

[14] A.V. Fursikov and O.Yu. Imanuvilov: On exact boundary zero controllability of the two-dimensional Navier-Stokes equation, Acta Appl. Math., 36, 1994, 1-10.

[15] A.V. Fursikov and O.Yu. Imanuvilov: Local exact controllability of the Navier-Stokes equations, RIM-GARC preprint series 95-92, Seoul National University, February 1996.

[16] G. Geymonat and E. Sanchez-Palencia: On the vanishing viscosity limit for acoustic phenomena in a bounded region, Arch. Rat. Mechanics and Analysis, 75, 1981, 257268.

[17] B.E. Launder and D.B. Spalding: Mathematical models of turbulence, Academic Press, 1972.

[18] J.-L. Lions: Quelques méthodes de résolution des problèmes aux limites non linéaires, Dunod et Gauthier-Villars, Paris, 1969.

[19] J.-L. Lions: Contrôle optimal de systèmes gouvernés par des équations aux dérivées partielles, Gauthier-Villars, Paris, 1968.

[20] J.-L. Lions: Are there connections between turbulence and controllability?, 9th INRIA International Conference, Antibes, June 12-25, 1990.

[21] J.-L. Lions: Exact controllability for distributed systems. Some trends and some problems, in: Applied and Industrial Mathematics, R. Spigler ed., Kluwer Academic Publishers, Dordrecht, Boston, London, 1991, 59-84.

[22] J.-L. Lions and E. Magenes: Problèmes aux limites non homogènes et applications, vol. 1, Dunod, Paris, 1968.

[23] P. Maremonti: Some theorems of existence for solutions of the Navier-Stokes equations with slip boundary condition in half-space, Ricerche di Matematica, 40, 1991, $81-135$.

[24] C.L.M.H. Navier: Sur les lois du mouvement des fluides, Mem. Acad. R. Sci. Inst. France, 6, 1823, 389-440.

[25] G.G. Stokes: On the effect of internal friction of fluids on the motion of pendulums, Trans. Cambridge Philos. Soc., 9, 1851, 8-106. 